\title{
Zur (Vor-)Wirkung und zu den Umsetzungsverpflichtungen von EU-Richtlinien - Eine unionsrechtliche Untersuchung der HETA-Abwicklung und des Moratoriums der österreichischen Finanzmarktaufsicht
}

\author{
Von Dr. Benedikt Pirker und Univ.-Prof. Dr. Astrid Epiney, Universität Freiburg i. Üe.
}

Dieser Beitrag wurde erstmals wie folgt veröffentlicht:

Benedikt Pirker/Astrid Epiney, Zur (Vor-)Wirkung und zu den Umsetzungsverpflichtungen von EURichtlinien - Eine unionsrechtliche Untersuchung der HETA-Abwicklung und des Moratoriums der österreichischen Finanzmarktaufsicht, VerwArch 2017, 304-342. Es ist möglich, dass die im VerwArch publizierte Version - die allein zitierfähig ist - im Verhältnis zu diesem Manuskript geringfügige Modifikationen enthält.

A. Einleitung und Problemstellung ....

B. Zur Anwendbarkeit der Abwicklungsrichtlinie und des BaSAG auf die Umwandlung der Hypo AlpeAdria-Bank International AG in eine Abbaueinheit

I. Zur Zulässigkeit der ausdrücklich geregelten Anwendbarkeit des BaSAG auf den Fall der Hypo AlpeAdria-Bank International AG

1. Zum Verhältnis der RL 2001/24/EG und der RL 2014/59/EU und zu den Vorgaben der RL 2014/59/EU.

a) Inhalt und Tragweite der RL 2001/24/EG und der RL 2014/59/EU - eine Skizze

b) Zum Verhältnis RL 2001/24/EG und der RL 2014/59/EU.

2. Zur Tragweite der „Vorwirkung“ der RL 2014/59/EU

3. Zu einem möglichen Verbot der zeitlich vorgezogenen Anwendung der eigenen Vorgaben in der RL 2014/59/EU......

4. Fazit

II. Zum persönlichen Anwendungsbereich der Abwicklungsrichtlinie 2014/59/EU

1. Zur Bedeutung und $\mathrm{zu}$ den Rechtsfolgen des Bestehens einer Verpflichtung aus der RL 2014/59/EU

2. Zum persönlichen Anwendungsbereich der RL 2014/59/EU und zum Begriff des Instituts
a) Auslegung gemäß dem Wortlaut
b) Teleologische Auslegung....
c) Systematische Auslegung.....
d) Folgenabschätzung einer engen Auslegung des Anwendungsbereichs und Sperrwirkung der RL 2014/59/EU
e) Zusätzliche Fragen des Anwendungsbereichs der RL 2014/59/EU

3. Zwischenergebnis

III. Zum sachlichen Anwendungsbereich der RL 2014/59/EU und der RL 2001/24/EG.....

1. Zum GSA als Abwicklungsmaßnahme und Vorwegnahme der Umsetzung der Richtlinie 2014/59/EU.

2. Zum GSA als Sanierungsmassnahme im Sinne der RL 2001/24/EG
a) Zum Verhältnis der Anwendungsbereiche der RL 2001/24/EG und der RL 2014/59/EU .......
aa) Zur These des Bedeutungsverlustes des Anwendungsbereichs von RL 2001/24/EG durch RL 2014/59/EU.....
bb) Zur These der Ergänzung des Anwendungsbereiches von RL 2001/24/EG durch RL 2014/59/EU.
cc) Zur These der Konkretisierung des Anwendungsbereiches von RL 2001/24/EG durch RL 2014/59/EU......
dd) Zwischenergebnis
b) Zum GSA und zum Begriff der Sanierungsmaßnahme im Sinne der RL 2001/24/EG nach alter Fassung. 
aa) Zum Begriff der „Sanierungsmaßnahme“ in der RL 2001/24/EG

bb) Zur Einstufung des GSA als „Sanierungsmaßnahme“

C. Zu den Rechtswirkungen des Moratoriums der FMA

I. Zur Einstufung des Moratoriums als Abwicklungsmaßnahme im Sinne der RL 2014/59/EU

II. Zur Einstufung des Moratoriums als Sanierungsmaßnahme im Sinne der RL 2001/24/EG

D. Zusammenfassung 


\section{A. Einleitung und Problemstellung ${ }^{1}$}

Die sog. Abwicklungsrichtlinie (RL 2014/59/EU, BRRD) ${ }^{2}$ enthält sowohl materiellrechtliche als auch prozessuale Vorgaben für die Sanierung und Abwicklung von näher definierten Kreditinstituten, wobei u.a. auch gewissen nationalen Maßnahmen transnationale Wirkung in anderen Mitgliedstaaten zukommen muss. In Österreich wurde die RL 2014/59/EU durch das Bundesgesetz über die Sanierung und Abwicklung von Banken (BaSAG) ${ }^{3}$ das am 1.1.2015 in Kraft getreten ist (womit die Umsetzungsfrist eingehalten wurde), ${ }^{4}$ umgesetzt. Im Vorfeld dieser Umsetzung durch das BaSAG wurde im Hinblick auf die Umwandlung der Hypo Alpe-Adria-Bank International AG (Firma später geändert in HETA) in eine Abbaueinheit im Sommer 2014 - also während der Frist für die Umsetzung der RL 2014/59/EU - das Bundesgesetz zur Schaffung einer Abbaueinheit (GSA) erlassen, in dessen Anwendung die Finanzmarktaufsicht mit (inzwischen rechtskräftigen Bescheid vom Herbst 2014) feststellte, dass diese Firma kein Einlagengeschäft mehr betrieb und keine qualifizierte Beteiligung an einem Kreditinstitut oder an einer Wertpapierfirma gehalten wurde, womit nach § 2 Abs. 3 GSA die Konzession zum Betrieb von Bankgeschäften endete. Obwohl die HETA damit zum Zeitpunkt des Inkrafttretens des BaSAG kein Kreditinstitut im Sinne dieses Gesetzes war, sieht $\S 162$ Abs. 6 BaSAG vor, dass der vierte Teile des Gesetzes - der Vorschriften zur Abwicklung enthält - auf die HETA anwendbar ist. ${ }^{5}$ Auf dieser Grundlage erliess die FMA im März 2015 per Bescheid ein Moratorium, mit dem mit sofortiger Wirkung der Fälligkeitszeitpunkt der vom Bescheid erfassten Verbindlichkeiten der HETA auf den 31.5.2016 hinausgeschoben wird.

Aus unionsrechtlicher Sicht wirft dieser Ablauf der Ereignisse bzw. das Vorgehen der österreichischen Behörden einige Fragen auf. Insbesondere ist zu klären, ob es unionsrechtlich zulässig oder gar geboten war, das BaSAG als Umsetzungsgesetz zur RL 2014/59/EU teilweise auf die HETA anzuwenden und welche Rechtsfolgen sich aus dieser Anwendung ergeben. Darüber hinaus ist als Folgefrage in Bezug auf das von der FMA im März 2015 per Bescheid erlassene Moratorium zu prüfen, ob das Moratorium als Maßnahme im Rahmen einer unionsrechtlich geregelten Abwicklungsbefugnis im Sinne der Sanierungsrichtlinie (RL 2001/24/EG ${ }^{6}$ ) in durch die RL 2014/59/EU geänderter Fassung einzustufen ist, da ihm als Folge auch unionsrechtlich geboten transnationale Wirkung etwa

$1 \quad$ Die vorliegende Untersuchung geht auf ein durch die Verfasser im Auftrag der Österreichischen Finanzmarktaufsicht erstelltes Gutachten zurück. Inhaltlich handelt es sich aber um ein unabhängiges Gutachten: Die Verfasser wurden ausdrücklich nicht auf eine vorgefasste Ansicht oder ein vorgegebenes Ergebnis verpflichtet, sondern um eine unabhängige Klärung der sich stellenden Fragen gebeten.

2 Als Banking Recovery and Resolution Directive bezeichnet, Richtlinie 2014/59/EU des Europäischen Parlaments und des Rates vom 15. Mai 2014 zur Festlegung eines Rahmens für die Sanierung und Abwicklung von Kreditinstituten und Wertpapierfirmen, ABl. 2014 L 173, 190 ff.

3 Bundesgesetz über die Sanierung und Abwicklung von Banken, BGBl I Nr. 98/2014.

4 S. Art. 130 Abs. 1 RL 2014/59, wonach die Richtlinie - mit Ausnahme einiger näher bezeichneter Bestimmungen - bis zum 31.12.2014 die Umsetzungsvorschriften zu erlassen und zu veröffentlichen haben; ab dem 1.1.2015 sind sie anzuwenden.

5 Vgl. zum Sachverhalt im Einzelnen, m.w.N., Perner, Zum rechtlichen Rahmen der HETAAbwicklung, Österreichisches BankArchiv 2015, S. 239 f. Siehe auch noch sogleich unten B., am Anfang.

6 In ihrer durch die BRRD abgeänderten Form, ursprünglich Richtlinie 2001/24/EG des Europäischen Parlaments und des Rates vom 4. April 2001 über die Sanierung und Liquidation von Kreditinstituten, ABl. 2001 L 125, 15 ff. 
vor Gerichten anderer Mitgliedstaaten zukäme, vor denen Gläubiger die Begleichung von unter das Moratorium fallenden Verbindlichkeiten einfordern. ${ }^{7}$

Vor diesem Hintergrund ergibt sich der Aufbau des vorliegenden Beitrags: In einem ersten Schritt ist die Anwendbarkeit der Abwicklungsrichtlinie auf die Umwandlung der Hypo AlpeAdria-Bank International AG in eine Abbaueinheit bzw. deren Verhältnis zum GSA und zum BaSAG zu prüfen (B.). Dabei ist insbesondere auf die ausdrücklich geregelte Anwendbarkeit des BaSAG auf den Fall der Hypo Alpe-Adria-Bank International AG und den Begriff des

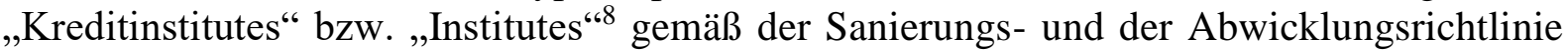
einzugehen, wobei sowohl danach gefragt wird, ob diese Anwendbarkeit zulässig ist als auch danach, ob sie gar geboten ist. Auf dieser Grundlage kann sodann analysiert werden, inwieweit das GSA als Abwicklungsmaßnahme im Sinne der Abwicklungsrichtlinie bzw. als Sanierungsmaßnahme im Sinne der Sanierungsrichtlinie eingeordnet werden kann, wobei es auch darum geht, ob das GSA auch als Maßnahme im Sinne der Abwicklungsrichtlinie bzw. als Vorwegnahme der Umsetzung der Abwicklungsrichtlinie angesehen werden kann. Ein eigener Abschnitt widmet sich den Rechtswirkungen des Moratoriums der FMA (C.). Dabei geht es insbesondere um die Einstufung des Moratoriums als unionsrechtlich geregelte Abwicklungs- oder Sanierungsmaßnahme im Sinne der Sanierungs- und der Abwicklungsrichtlinie und zur daraus resultierenden unionsrechtlichen Verpflichtung zur Anerkennung seiner Rechtswirkung für die Gerichte anderer Mitgliedstaaten. Die wesentlichen Ergebnisse des Beitrags werden schliesslich in Thesenform zusammengefasst (D.).

Auch wenn der Ausgangspunkt des vorliegenden Beitrags Fragen sind, die sich im Zusammenhang mit der konkreten, soeben skizzierten Situation bzw. den entsprechenden österreichischen Maßnahmen gestellt haben, ist nicht $\mathrm{zu}$ verkennen, dass sie auch grundlegende Fragen des Unionsrechts berühren, so insbesondere solche nach den genauen Rechtswirkungen von Richtlinien und nach den sog. Vorwirkungen einer Richtlinie (also m.a.W. die sich aus ihr ergebenden Verpflichtungen während der Umsetzungsfrist). Insofern ist der vorliegende Beitrag auch über die konkrete Fragestellung hinaus von einem gewissen Interesse und auch für andere Rechtsgebiete grundsätzlich relevant.

\section{B. Zur Anwendbarkeit der Abwicklungsrichtlinie und des BaSAG auf die Umwandlung der Hypo Alpe-Adria-Bank International AG in eine Abbaueinheit}

Ausgehend von der bereits eingangs kurz skizzierten Ausgangslage geht es nachfolgend um die Analyse derjenigen unionsrechtlichen Vorgaben, die im Zusammenhang mit dem GSA sowie der ausdrücklich geregelten Anwendbarkeit des BaSAG auf die HETA relevant sein könnten und insbesondere auch Aufschluss über die unionsrechtliche Zulässigkeit bzw. Gebotenheit des Vorgehens geben könnten. Im Einzelnen ist insbesondere der Anwendungsbereich der RL 2001/24/EG und 2014/59/EU zu klären, woraus sich dann auch

$7 \quad$ Andere Maßnahmen im österreichischen Recht wie etwa das angeordnete Erlöschen gewisser nicht strittiger bzw. die Stundung gewisser strittiger Sanierungsverbindlichkeiten zu prüfen, würde hingegen den Rahmen des vorliegenden Beitrags sprengen. Siehe hierzu näher Fest, Abwicklung der Hypo Alpe-Adria-Bank: Keine Anerkennung der Maßnahmen ausserhalb des Herkunftsstaates auf Grundlage der Sanierungs-RL und der BRRD, NZG 2015, S. 1108 ff. (S. 1109).

$8 \quad$ In der Richtlinie 2014/59/EU werden auch Regelungen in Bezug auf Wertpapierfirmen getroffen. Der Einfachheit halber wird in der folgenden Untersuchung regelmäßig nur der schwerpunktmäßig interessantere Fall der Kreditinstitute angesprochen. 
ergibt, ob die RL 2014/59/EU auf die HETA anwendbar ist. Weiter fragt es sich, ob sich das GSA als Maßnahme auf der Grundlage dieser Richtlinie bzw. als Vorwegnahme der Umsetzung einordnen lässt und ob das GSA alternativ dazu oder gleichzeitig eine Maßnahme im Sinne der RL 2001/24/EG darstellt.

Damit ist in einem ersten Schritt (I.) der Frage nachzugehen, ob der österreichische Gesetzgeber mit der vorzeitigen Anwendung der Vorgaben der RL 2014/59/EU aus unionsrechtlicher Sicht zulässig gehandelt hat. Danach ist der persönliche Anwendungsbereich der RL 2014/59/EU daraufhin zu prüfen, ob er sich auch auf einen Fall wie jenen der Schaffung der HETA als Abbaueinheit erstreckt (II.). In einem solchen Fall wäre nämlich der österreichische Gesetzgeber zu seinem Vorgehen sogar unionsrechtlich verpflichtet. Schliesslich ist auf den sachlichen Anwendungsbereich der RL 2014/59/EU und 2001/24/EG einzugehen, um zu eruieren, ob das GSA in den jeweiligen Anwendungsbereich einer der beiden oder gar beider Richtlinien einzuordnen ist (III.).

Um die damalige Hypo Alpe-Adria-Bank International AG in eine Abbaueinheit umzuwandeln, erliess der österreichische Gesetzgeber das Bundesgesetz zur Schaffung einer Abbaueinheit (GSA), ${ }^{9}$ das am 1. August 2014 in Kraft trat. Gemäß $\S 2$ Abs. 1 GSA sollte die Hypo Alpe-Adria-Bank International AG kein Einlagengeschäft ${ }^{10}$ mehr betreiben und keine qualifizierte Beteiligung an einem Kreditinstitut oder an einer Wertpapierfirma mehr halten und die FMA dies mittels Bescheid feststellen. Ab der Rechtskraft dieses Bescheides sollte die der Hypo Alpe-Adria-Bank International AG aufgrund des Bankwesengesetzes (BWG) erteilte Konzession für den Betrieb von Bankgeschäften enden und letztere als reine Abbaueinheit mit der primären Aufgabe des Portfolioabbaus weitergeführt werden ( 3 Abs. 1 GSA).

$\mathrm{Zu}$ diesem Zweck veräußerte im September und Oktober 2014 die Hypo Alpe-Adria-Bank International AG ihre Aktien an der Hypo SEE Holding AG an die FIMBAG Finanzmarktbeteiligung AG des Bundes und ihre Aktien an der Hypo Alpe-Adria Bank S.p.A. an die HBI-Bundesholding AG. Darüber hinaus zeigte der Vorstand der FMA an, dass die Hypo Alpe-Adria-Bank International AG kein Einlagengeschäft mehr betreibe, was vom Bankprüfer bestätigt wurde. Zuletzt wurde am 30. Oktober 2014 die Firma der Hypo Alpe-Adria-Bank International AG auf HETA Asset Resolution AG (HETA) geändert und die Aufgabe der Gesellschaft den Vorgaben des GSA angepasst.

Daraufhin erliess die FMA, wie im GSA vorgesehen, am 30.10.2014 einen mittlerweile in Rechtskraft erwachsenen Bescheid, gemäß welchem die Konzession zum Betrieb von Bankgeschäften der Hypo AlpeAdria-Bank International AG endete, da die Voraussetzungen des $§ 2$ Abs. 1 GSA erfüllt waren, und die Hypo Alpe-Adria-Bank International AG als Abbaueinheit im Sinne des $§ 3$ GSA fortgeführt werde.

Am 1. Januar 2015 trat darüber hinaus das Bundesgesetz über die Sanierung und Abwicklung von Banken (BaSAG) in Kraft, mit dem die RL 2014/59/EU zur Sanierung und Abwicklung von Kreditinstituten in das österreichische Recht umgesetzt wurde. § 162 Abs. 6 BaSAG erklärt den 4. Teil desselben Gesetzes, d.h. die Vorschriften zur Abwicklung, auch auf die auf der Grundlage von $§ 2$ GSA geschaffene Abbaueinheit für anwendbar. ${ }^{11}$

\section{Zur Zulässigkeit der ausdrücklich geregelten Anwendbarkeit des BaSAG auf den Fall der Hypo Alpe-Adria-Bank International AG}

In einem ersten Schritt ist die Zulässigkeit des Vorgehens des österreichischen Gesetzgebers zu prüfen, das BaSAG auf die Hypo Alpe-Adria-Bank International AG bzw. die HETA für anwendbar zu erklären und damit die Möglichkeit für deren Umwandlung in eine Abbaueinheit durch das GSA vorzusehen.

Als Ausgangspunkt für die Beantwortung dieser Frage ist festzuhalten, dass der österreichische Gesetzgeber mit dem Erlass des BaSAG die Verpflichtungen aus der RL 2014/59/EU in das österreichische Recht umsetzen wollte. Dieser - soweit ersichtlich unbestrittene - Charakter des BaSAG als Umsetzungsgesetz zur RL 2014/59/EU ergibt sich

$9 \quad$ BGBl I Nr. 51/2014.

10 Gemäß $§ 1$ Abs. 1 Ziff. 1 Bankwesengesetz (BWG).

11 Siehe insgesamt auch die Übersicht über den Sachverhalt bei Perner (Fußn. 5), S. 239. 
bereits aus dem entsprechenden Umsetzungshinweis in Artikel 1 BaSAG. In Bezug auf die Umsetzungsfrist_sieht die RL 2014/59/EU vor, dass bis zum 31. Dezember 2014 die entsprechenden Rechts- und Verwaltungsvorschriften zur Umsetzung der Richtlinie erlassen werden und ab dem 1. Januar 2015 angewendet werden müssen. ${ }^{12}$ Durch $§ 162$ Abs. 6 BaSAG erklärt der österreichische Gesetzgeber die im vierten Teil desselben Gesetzes vorgesehenen Befugnisse und Instrumente auf die im Rahmen des GSA vor Ablauf der Umsetzungsfrist und vor Inkrafttreten des BaSAG geschaffene Abbaueinheit anwendbar. Eine derart rückwirkende Anwendung der Umsetzungsvorschriften auf eine bereits zuvor geschaffene Abbaueinheit könnte über die Vorgaben der Richtlinie hinausgehen und gar mit sich aus dieser ergebenden Verpflichtungen unvereinbar sein. Deutlich wird damit, dass es im vorliegenden Zusammenhang letztlich um die Frage geht, ob es aus unionsrechtlicher Sicht zulässig ist, gewisse sich aus der RL 2014/59/EU ergebende Vorgaben auch auf eine Konstellation wie die vorliegende anzuwenden, die auf Umständen beruht, die vor dem Ablauf der Umsetzungsfrist angesiedelt sind. M.a.W. steht im Ergebnis die Zulässigkeit der „Vorwirkung“ der entsprechenden Richtlinienbestimmungen in der hier zur Debatte stehenden Situation in Frage. Zu prüfen ist somit, ob sich aus dem Unionsrecht ergibt, dass eine solche durch das nationale Recht gewählte Ausdehnung des zeitlichen Anwendungsbereichs gewisser Vorgaben der RL 2014/59/EU unionsrechtlich untersagt ist. Hierfür ist einleitend das Verhältnis der RL 2001/24/EG und der RL 2014/59/EU zu erörtern, könnte sich doch aus der rückwirkenden Anwendung der Vorgaben der späteren Richtlinie vor Ablauf der Umsetzungsfrist ein inhaltlicher Konflikt mit den Vorgaben der früheren Richtlinie ergeben (1.). Danach ist zu prüfen, ob sich aus der Vorwirkung der RL 2014/59/EU (2.) oder aus deren Bestimmungen selbst (3.) die Unzulässigkeit des erwähnten Vorgehens ergeben könnte, bevor ein kurzes Fazit gezogen werden kann (4.).

\section{Zum Verhältnis der RL 2001/24/EG und der RL 2014/59/EU und zu den Vorgaben der RL 2014/59/EU}

Bis zum Ablauf der Umsetzungsfrist der RL 2014/59/EU waren bzw. sind die Mitgliedstaaten verpflichtet, die einschlägigen bis dahin geltenden unionsrechtlichen Vorgaben zu beachten und anzuwenden. Damit wird die Frage aufgeworfen, ob aufgrund der erwähnten Bestimmung des BaSAG bzw. der Anwendung des GSA zur Schaffung der HETA Normen des nationalen Rechts zur Anwendung kommen, die nicht im Einklang mit den bis zum Ablauf der Umsetzungsfrist der RL 2014/59/EU geltenden unionsrechtlichen Vorgaben stehen, dies vor dem Hintergrund, dass gewisse Teile der Umsetzungsgesetzgebung auf Ereignisse zur Anwendung kommen, die vor Ablauf dieser Frist stattfanden. Für die Rechtslage bis zum 31.12.2014 sind die Normen der Sanierungsrichtlinie (RL 2001/24/EG) als Vorgängerrichtlinie massgeblich, womit auch die Frage nach dem Verhältnis der RL 2001/24/EG und der RL 2014/59/EU aufgeworfen wird (b), eine Fragestellung, der ausgehend von einigen einleitenden Bemerkungen zum Inhalt der beiden Rechtsakte nachzugehen ist (a).

\section{a) Inhalt und Tragweite der RL 2001/24/EG und der RL 2014/59/EU - eine Skizze}

Die RL 2001/24/EG schafft Sonderregelungen für die Sanierung und Liquidation von Kreditinstituten mit Hauptsitz bzw. Zweigniederlassungen im Europäischen Wirtschaftsraum. Ziel ist es, im Interesse von Gläubigern die Aufsichtsbehörden in das 
Zwangsliquidationsverfahren einzubinden und zur Vermeidung von Kompetenzkonflikten und Ungleichbehandlungen sicherzustellen, dass nur ein einziges Liquidationsverfahren mit unionsweiter Wirkung durchgeführt wird. ${ }^{13}$ Die Richtlinie ist auf Kreditinstitute anwendbar, wobei dieser Begriff mit Verweis auf RL 2000/12/EG ${ }^{14}$ definiert wird. ${ }^{15}$ Demzufolge handelt es sich um ein Kreditinstitut bei einem „Unternehmen, dessen Tätigkeit darin besteht, Einlagen oder andere rückzahlbare Gelder des Publikums entgegenzunehmen und Kredite für eigene Rechnung zu gewähren“. ${ }^{16} \mathrm{Zu}$ Zwecken der Beaufsichtigung von Grosskrediten werden in diese Definition auch Zweigniederlassungen miteinbezogen. ${ }^{17}$

Im Verhältnis zu diesem, zwar teils unionsrechtlich geregelten, jedoch letztlich „,regulären“ Insolvenzverfahren haben die einschlägigen Vorgaben der RL 2014/59/EU zur Abwicklung Vorrang, wenn es zu einem Abwicklungsverfahren kommt. ${ }^{18}$ Ein solches Verfahren kommt nach Art. 32 und 33 der RL 2014/59/EU dann zur Anwendung, wenn der Ausfall eines Kreditinstituts durch Maßnahmen der Privatwirtschaft oder der Aufsichtsbehörden ${ }^{19}$ innerhalb eines angemessenen Zeitrahmens wahrscheinlich nicht abgewendet werden kann und wenn ein öffentliches Interesse an der Abwicklung besteht. ${ }^{20}$ Ein solches Interesse wiederum besteht, wenn eine Abwicklungsmaßnahme für das Erreichen mindestens eines Abwicklungsziels ${ }^{21}$ der Richtlinie erforderlich, mit Blick auf dieses Ziel hin verhältnismäßig ist und dies bei einer Liquidation auf dem Wege eines regulären Insolvenzverfahrens nicht in demselben Umfang der Fall wäre. ${ }^{22}$

Im Abwicklungsverfahren ${ }^{23}$ gemäß der RL 2014/59/EU hat die Abwicklungsbehörde darauf zu achten, dass die Verluste primär von den Anteilseignern und sekundär von den Gläubigern getragen werden ${ }^{24}$ und die Gläubiger keine grösseren Verluste erleiden als im Wege eines regulären Insolvenzverfahrens. ${ }^{25}$ Mindestens vier Abwicklungsinstrumente müssen von den Mitgliedstaaten im nationalen Recht vorgesehen werden: ${ }^{26}$

- Unternehmensveräußerungen an Dritte, die kein Brückeninstitut sind, ${ }^{27}$

- Unternehmensveräußerungen an ein Brückeninstitut, das von der Abwicklungsbehörde kontrolliert wird und deren Leitungsorgane und Geschäftsleiter maximal für grobe Fahrlässigkeit haften; ${ }^{28}$

13 Jung/Bischof, Europäisches Finanzmarktrecht - Die Regulierung von Märkten, Unternehmen und Dienstleistungen durch die Europäische Union, Baden-Baden 2015, Rdnr. 166.

14 AB1. 2000 L126, $1 \mathrm{ff}$.

15 Art. 1 Abs. 1 RL 2001/24/EG.

16 Art. 1 Abs. 1 erster Satz RL 2000/12/EG.

17 Art. 1 Abs. 1 dritter Satz RL 2000/12/EG.

18 Siehe Art. 37 Abs. 6 RL 2014/59/EU, der regelt, dass nur jene Teile der Vermögenswerte, Rechte oder Verbindlichkeiten eines in Abwicklung befindlichen Instituts, die verbleiben, im Rahmen eines regulären Insolvenzverfahrens liquidiert werden.

19 Etwa durch Frühinterventionsmaßnahmen nach Art. 27 RL 2014/59/EU.

$20 \quad$ Jung/Bischof (Fußn. 13), Rdnr. 163.

21 Art. 31 Abs. 2 RL 2014/59/EU.

$22 \quad$ Art. 32 Abs. 5 RL 2014/59/EU.

23 Siehe zu den Eckpunkten des Verfahrensablaufes Huhtaniemi/Nava/Tornese, The Establishment of a EU-Wide Framework for the Resolution of Banks and Financial Institutions, in: HinojosaMartínez/Beneyto (Hrsg.), European Banking Union - The New Regime, Alphen aan den Rijn 2015, S. 99 ff. (S. 104). Art. 34 Abs. 1 RL 2014/59/EU. Art. 73-75 RL 2014/59/EU.

Art. 37 Abs. 1 und 3 RL 2014/59/EU. Vgl. auch zur Interaktion dieser Instrumente Huhtaniemi et. al. (Fußn. 23), S. $105 \mathrm{f}$.

Art. 38 und 39 RL 2014/59/EU.

Art. 40 und 41 RL 2014/59/EU. 
- die Ausgliederung von Vermögenswerten, Rechten und Verbindlichkeiten auf eine Zweckgesellschaft (sogenannte Bad Bank); ${ }^{29}$ und

- $\quad$ ein Bail-in-Instrument, mit dem die Abwicklungsbehörde Verbindlichkeiten in einer vorgegebenen Reihenfolge ${ }^{30}$ herabschreiben und/oder in Eigenkapital umwandeln kann, um das Institut $\mathrm{zu}$ rekapitalisieren oder andere Abwicklungsmaßnahmen zu ermöglichen. ${ }^{31}$

Schliesslich enthält die Richtlinie auch Vorgaben zur Kooperation zwischen Behörden verschiedener Mitgliedstaaten bei grenzüberschreitenden Gruppen von Kreditinstituten, etwa in Bezug auf den Informationsaustausch und gegenseitige Konsultationen. ${ }^{32}$

Die RL 2014/59/EU ist Teil der sogenannten zweiten Säule der Bankenunion, die sich aus den drei Säulen eines einheitlichen Aufsichtsmechanismus ', eines einheitlichen Abwicklungsmechanismus ' und einer harmonisierten dezentralen Einlagensicherung zusammensetzt bzw. zusammensetzen soll. ${ }^{33}$ Sie fügt sich somit in einen komplexen rechtlichen Rahmen innerhalb und teils außerhalb des Unionsrechts ein ${ }^{34}$ und setzt gleichzeitig die Vorgaben und Grundsätze internationaler Fachgremien, wie etwa des Financial Stability Forum, um. ${ }^{35}$

Gewisse Aspekte der RL 2014/59/EU sind auch teilweise auf Kritik gestoßen: Aufgrund ihres dezentralen Vorgehens, das nur gemeinsame Auflösungsmechanismen vorgibt, bleibe das Risiko einander widersprechender Entscheidungen in verschiedenen Mitgliedstaaten grundsätzlich bestehen. Darüber hinaus änderten auch die Finanzierungsvorgaben für Abwicklungsfonds nichts daran, dass Banken erneut zum Risiko bzw. abhängig von Unterstützung aus den Haushalten der Mitgliedstaaten werden könnten. ${ }^{36}$

\section{b) Zum Verhältnis RL 2001/24/EG und der RL 2014/59/EU}

Soweit das Verhältnis zwischen den beiden Richtlinien betroffen ist, sieht der durch die RL 2014/59/EU in die RL 2001/24/EG eingefügte Art. 1 Abs. $4^{37}$ vor, dass im Fall einer Anwendung der Abwicklungsinstrumente und einer Ausübung der Abwicklungsbefugnisse gemäß der RL 2014/59/EU die RL 2001/24/EG auch für die in den Anwendungsbereich der RL 2014/59/EU fallenden Finanzinstitute, Firmen und Mutterunternehmen gilt. Werden also Abwicklungsmaßnahmen im Sinne der RL 2014/59/EU auf Kreditinstitute angewendet, gilt auch die RL 2001/24/EG.

Darüber hinaus ergänzt die RL 2014/59/EU durch eine Abänderung von Art. 2 siebter Spiegelstrich RL 2001/24/EG die Legaldefinition des Begriffs der „Sanierungsmaßnahmen“ dahingehend, dass als solche Maßnahmen auch die Anwendung der Abwicklungsinstrumente und Ausübung der Abwicklungsbefugnisse aus der RL 2014/59/EU zu verstehen sind. ${ }^{38}$

29 Art. 42 RL 2014/59/EU.

30 Vgl. hierzu auch Huhtaniemi et. al. (Fußn. 23), S. 104.

31 Art. 43, 44 und 48 RL 2014/59/EU.

32 Hierzu Huhtaniemi et. al. (Fußn. 23), S. $106 \mathrm{f}$.

33 Jung/Bischof (Fußn. 13), Rdnr. 7. Für einen geschichtlichen Überblick über die Entstehung der Richtlinie siehe Huhtaniemi et. al. (Fußn. 23), S. 102 f.

34 Siehe etwa zur Schwierigkeit, die genauen Verbindungen zwischen der Abwicklungsrichtlinie und dem völkerrechtlichen Abkommen zum Mechanismus zur Bankenabwicklung auszuloten Moloney, European Banking Union: Assessing Its Risks and Resilience, Common Markt Law Review 2014, S. 1609 ff. (S. 1626).

Lopez-Escudero, EU Banking Union and International Financial Law, in: HinojosaMartínez/Beneyto (Hrsg.), European Banking Union - The New Regime, Alphen aan den Rijn 2015, S. 179 ff. (S. 205); Huhtaniemi et. al. (Fußn. 23), S. 101. Lopez-Escudero (Fußn. 35), S. 204.

Art. 117 Abs. 1 RL 2014/59/EU.

Art. 117 Abs. 2 RL 2014/59/EU. 
Auch in Bezug auf ihre Zielsetzung stehen die beiden Richtlinien nicht im Widerspruch zueinander, sondern sollen sich ergänzen und in ihrer Wirkung gegenseitig verstärken. Dies kommt in der Zielsetzung der RL 2014/59/EU deutlich zum Ausdruck, indem eine wirksame Abwicklung dadurch sichergestellt werden soll, dass die RL 2001/24/EG und die damit verbundene gegenseitige Anerkennung und der Vollzug von Beschlüssen in Bezug auf Sanierungen und Liquidationen von Kreditinstituten ${ }^{39}$ in verschiedenen Mitgliedstaaten auch beim Rückgriff auf Abwicklungsinstrumente der RL 2014/59/EU zur Geltung kommen. ${ }^{40}$ Deutlich wird damit, dass die RL 2001/24/EG keine strengeren oder die Neuerungen der RL 2014/59/EU ausschließenden Vorgaben enthält; vielmehr führte die RL 2014/59/EU lediglich zur Ergänzung der RL 2001/24/EG und einer damit einhergehenden Erweiterung ihres Anwendungsbereichs. Daher kann ausgeschlossen werden, dass die rückwirkende Anwendung der Abwicklungsvorgaben des BaSAG auf die durch das GSA geschaffene Abbaueinheit im Widerspruch zu den vor Ablauf der Umsetzungsfrist der RL 2014/59/EU geltenden Vorschriften steht.

\section{Zur Tragweite der „Vorwirkung“ der RL 2014/59/EU}

Während der Umsetzungsfrist von Richtlinien dürfen grundsätzlich keine Vorschriften erlassen oder Maßnahmen ergriffen werden, welche die Erreichung der Zielsetzungen der betreffenden Richtlinie ernstlich in Frage stellen bzw. gefährden können. ${ }^{41}$ Hieraus leitet sich zwar keine allumfassende Sperrwirkung ab, jedoch ein „Frustrationsverbot“, das sich auf die Erreichung der Richtlinienziele bezieht. ${ }^{42}$

In unserem Zusammenhang stellt sich daher die Frage, ob das skizzierte Vorgehen des österreichischen Gesetzgebers gegen diese unionsrechtlichen Pflichten verstieß. Dies ist im Ergebnis zu verneinen: Im vorliegenden Fall liegt schon keine Gefährdung der Erreichung der Ziele der RL 2014/59/EU vor. Diese zielt auf die Schaffung eines glaubwürdigen Sanierungs- und Abwicklungsrahmens für den wirksamen Umgang mit unsoliden oder ausfallenden Kreditinstituten und Wertpapierfirmen ab. ${ }^{43}$ Die reine zeitliche Erstreckung der Anwendung der ins nationale Recht umgesetzten Vorgaben der Richtlinie auf einen Sachverhalt, der seinen Anfang vor Ablauf der Umsetzungsfrist nahm, steht mit diesen Zielsetzungen nicht im Widerspruch, im Gegenteil: Eine solche Erstreckung fördert sogar das Erreichen der Ziele der Richtlinie. So kommen nämlich durch das GSA inhaltliche Vorgaben zur Abwicklung im vorliegenden Fall bereits auf die HETA als Abbaueinheit zur Anwendung, wenngleich die Konzession für den Betrieb von Bankgeschäften bereits vor Ablauf der Umsetzungsfrist bzw. vor Inkrafttreten des BaSAG endete. ${ }^{44}$

\footnotetext{
39 Siehe hierzu noch B.II.1.

$40 \quad$ Erw. 119 RL 2014/59/EU.

41 EuGH, Rs. C-129/96 (Inter-Environnement Wallonie), EU:C:1997:628, Rdnr. 45. Diese Grundsätze bestätigend z.B. EuGH, RS. C-422/05 (Kommission/Belgien), EU:C:2007:342; EuGH, Rs. C138/05 (Stiching Zid-Hollandse), EU:C:2006:577.

42 Kühling, Vorwirkungen von EG-Richtlinien bei der Anwendung nationalen Rechts Interpretationsfreiheit für Judikative und Exekutive?, DVBl 2006, S. 857 ff. (S. 858).

43 Siehe Erw. 1 RL 2014/59/EU.

$44 \quad$ Hingegen treten die Rechtswirkungen des Unionsrechts, d.h. der Richtlinie 2014/59/EU, effektiv erst ab dem Ablauf der Umsetzungsfrist der Richtlinie hinzu, siehe hierzu noch näher unten B.II.1.
} 


\section{Zu einem möglichen Verbot der zeitlich vorgezogenen Anwendung der eigenen Vorgaben in der RL 2014/59/EU}

Schließlich ist noch danach zu fragen, ob die RL 2014/59/EU selbst Bestimmungen enthält, die eine zeitlich vorgezogene Anwendung ihrer Vorgaben durch nationales Recht auf vor Ablauf der Umsetzungsfrist entstandene Sachverhalte untersagt.

Anhaltspunkte hierfür sind jedoch keine ersichlich, im Gegenteil: Art. 1 Abs. 2 RL 2014/59/EU hält ausdrücklich fest, dass die Richtlinie Mindestvorschriften enthält, so dass die Mitgliedstaaten strengere nationale Vorschriften vorsehen dürfen. ${ }^{45}$ Gemäß dem Wortlaut der Bestimmung können die Mitgliedstaaten strengere Bestimmungen nicht nur „erlassen“, sondern auch „,beibehalten“, solange kein Widerspruch zur Richtlinie entsteht. Damit wäre sogar eine Umsetzung strengerer Vorgaben als jener der Richtlinie vor Ablauf der Umsetzungsfrist letzterer zulässig. Die weniger weitreichende Anwendung von nationalen Umsetzungsvorschriften auf vor Ablauf der Umsetzungsfrist erfolgte Ereignisse ist somit jedenfalls auch als zulässig einzustufen.

\section{4. $\quad$ Fazit}

Insgesamt ergibt sich somit, dass der österreichische Gesetzgeber zwar (auf den ersten Blick) über die sich aus der RL 2014/59/EU ergebenden Verpflichtungen hinausging, indem er durch das GSA die Möglichkeit der Schaffung einer Abbaueinheit einrichtete und das BaSAG ausdrücklich auf die HETA als Abbaueinheit anwendbar erklärte. Jedoch widerspricht ein solches Vorgehen nicht dem Unionsrecht, weder der vor Ablauf der Umsetzungsfrist der RL 2014/59/EU geltenden Rechtslage, noch den Verpflichtungen vor Ablauf der Umsetzungsfrist der Richtlinie, noch den Bestimmungen der Richtlinie selbst.

\section{Zum persönlichen Anwendungsbereich der RL 2014/59/EU}

Die bislang angestellten Überlegungen lassen erkennen, dass das Unionsrecht dem österreichischen Gesetzgeber jedenfalls den notwendigen Spielraum einräumt, um durch das GSA und später das BaSAG gewisse Vorgaben des BaSAG bzw. der RL 2014/59/EU auf die HETA als bereits vor Ablauf der Umsetzungsfrist errichtete Abbaueinheit anzuwenden. Auf dieser Grundlage und daran anschließend fragt es sich, ob sich aus dem Unionsrecht nicht gar eine Verpflichtung zu einem solchen Vorgehen ableiten lässt, ob also m.a.W. eine Pflicht bestand, das BaSAG auf die HETA als Abbaueinheit für anwendbar zu erklären. Bevor hierfür der persönliche Anwendungsbereich der RL 2014/59/EU näher geprüft wird (2.), bedürfen Bedeutung und Auswirkungen des Bestehens einer solchen Verpflichtung näherer Erläuterung (1.).

\section{Zur Bedeutung und zu den Rechtsfolgen des Bestehens einer Verpflichtung aus der RL 2014/59/EU}


Im vorliegenden Zusammenhang ergäben sich aus dem Bestehen einer Verpflichtung des österreichischen Gesetzgebers, die HETA als Abbaueinheit in den Anwendungsbereich der Umsetzungsgesetzgebung zur RL 2014/59/EU und damit des BaSAG einzubeziehen, besondere Folgen. Geht man nämlich davon aus, dass für den österreichischen Gesetzgeber lediglich kein unionsrechtliches Verbot eines solchen Vorgehens bestand, d.h. sein Vorgehen erlaubt, aber nicht geboten war, ist bzw. wäre die Schaffung der HETA durch das GSA bzw. der Einbezug der HETA in den Anwendungsbereich des BaSAG - soweit nicht die RL 2001/24/EG anwendbar ist ${ }^{46}$ - nicht näher unionsrechtlich determiniert und nach österreichischem Recht zu beurteilen; damit gilt bzw. gälte für die auf dieser Basis getroffenen Abwicklungsmaßnahmen nur das österreichische Recht. Dieses kann jedoch nicht die Anerkennung von Abwicklungsmaßnahmen in anderen EU-Mitgliedstaaten anordnen. Fällt hingegen der Einbezug der HETA in das BaSAG unter die Verpflichtungen aus der RL 2014/59/EU, kann sich daraus auch eine unionsrechtlich begründete Verpflichtung zur Anerkennung von somit unionsrechtlich geregelten Abwicklungsmaßnahmen in den anderen Mitgliedstaaten ergeben. ${ }^{47}$

Bereits in der RL 2001/24/EG, die sich mit dem anwendbaren Recht und der Zuständigkeit bei grenzüberschreitenden Sanierungen und Insolvenzen von Kreditinstitutionen befasste, verfolgte der Unionsgesetzgeber das Ziel, ein einheitliches Insolvenzverfahren für derartige Fälle zu schaffen, wofür auch die gegenseitige Anerkennung von Maßnahmen eines Mitgliedstaates erforderlich sei. ${ }^{48}$ Insbesondere sieht Art. 3 Abs. 2 RL 2001/24/EG betreffend das anwendbare Recht vor, dass das Recht des Herkunftsmitgliedstaates für Sanierungsmaßnahmen gilt und dass diese Maßnahmen in der gesamten Union „ohne weitere Formalität uneingeschränkt wirksam" sind, was auch Dritte in anderen Mitgliedstaaten oder Situationen betrifft, in denen im Recht eines anderen Mitgliedstaates solche Maßnahmen nicht vorgesehen sind oder Voraussetzungen für deren Durchführung nicht erfüllt sind. Darüber hinaus gilt gemäß Art. 9 Abs. 1 S. 2 RL 2001/24/EG eine Entscheidung zur Eröffnung eines Liquidationsverfahrens durch die zuständige Behörde eines Mitgliedstaates „ohne weitere Formalität“ in allen Mitgliedstaaten als ,,anerkannt“ und „wirksam“.

Die RL 2014/59/EU ändert diesbezüglich, wie erläutert, ${ }^{49}$ den Anwendungsbereich der RL 2001/24/EG. ${ }^{50}$ Werden Abwicklungsmaßnahmen im Sinne der RL 2014/59/EU auf Institute angewendet, gilt daher auch die RL 2001/24/EG und somit eine unionsrechtlich verbürgte Verpflichtung der anderen Mitgliedstaaten, solche Abwicklungsmaßnahmen anzuerkennen und ihnen Wirksamkeit in der eigenen Rechtsordnung zukommen zu lassen. Allerdings gilt diese Erstreckung der Rechtswirkungen aus der RL 2001/24/EG erst ab dem Zeitpunkt des Ablaufes der Umsetzungsfrist der RL 2014/59/EU, da diese erst ab jenem Zeitpunkt volle Wirksamkeit erlangt bzw. im nationalen Recht umzusetzen ist. Erst ab diesem Zeitpunkt werden somit Abwicklungsmaßnahmen, die womöglich andauern bzw. derartige Maßnahmen, die danach getroffen werden, voll wirksam im Sinne der RL 2001/24/EG, was insbesondere eine Verpflichtung zur Anerkennung in anderen Mitgliedstaaten mit sich bringt. Somit wird die Bedeutung der Fragestellung klar, ob die Anwendung der Richtlinienvorgaben, wie sie durch das GSA erfolgt bzw. im BaSAG für den Fall der HETA als Abbaueinheit, die vor dem Ablauf der Umsetzungsfrist der RL 2014/59/EU geschaffen wurde, vorgesehen ist, lediglich unionsrechtlich erlaubt oder aber unionsrechtlich als Teil der

\footnotetext{
$46 \quad$ Hierzu noch näher unten B.III.

$47 \quad$ So überzeugend Perner (Fußn. 5), S. 241.

48 So etwa Erw. 7 RL 2001/24/EG.

49 Siehe B.I.1.

50 Art. 117 Abs. 1 RL 2014/59/EU.
} 
Umsetzung der RL 2014/59/EU geboten ist. Im ersten Fall gelten Abwicklungsmaßnahmen in Bezug auf die HETA rein gemäß österreichischem Recht, soweit nicht aus anderen Gründen die RL 2001/24/EG zur Anwendung kommt, ${ }^{51}$ und kommen solchen Maßnahmen in der Rechtsordnung anderer Mitgliedstaaten nur Rechtswirkungen zu, soweit dies im dortigen nationalen Recht vorgesehen ist. Im zweiten Fall hingegen entsteht eine unionsrechtliche Verpflichtung, solche Abwicklungsmaßnahmen im nationalen Recht sämtlicher Mitgliedstaaten anzuerkennen und ihnen vollumfänglich Wirkung zuzuerkennen, was etwa die Anerkennung des - am 1. März 2015 und somit nach Ablauf der Umsetzungsfrist der RL 2014/59/EU erlassenen - Moratoriums der österreichischen FMA betreffen könnte. ${ }^{52}$

\section{Zum persönlichen Anwendungsbereich der RL 2014/59/EU und zum Begriff des Instituts}

Die RL 2014/59/EU findet auf Institute im Sinne der Richtlinie Anwendung. Damit ist im vorliegenden Zusammenhang entscheidend, ob die RL 2014/59/EU auch auf Institute anzuwenden ist, die wie die Abbaueinheit ihre Zulassung zum Zeitpunkt des Ablaufens der Umsetzungsfrist der RL 2014/59/EU bereits verloren haben, so dass es letztlich um die Auslegung des Begriffs des Instituts im Sinne der RL 2014/59/EU geht. Für die somit erforderliche Auslegung der einschlägigen Bestimmungen der Richtlinie sind die verschiedenen Auslegungsmethoden, wie sie im Unionsrecht Anwendung finden, heranzuziehen.

\section{a) Auslegung gemäß dem Wortlaut}

Nach Art. 1 Abs. 1 Bst. a RL 2014/59/EU schafft die Richtlinie Vorgaben für die Sanierung und Abwicklung von „Instituten“",53 die in der Union niedergelassen sind. Gemäß Art. 2 Abs. 1 Ziff. 23 RL 2014/59/EU bezeichnet der Begriff des Instituts „ein Kreditinstitut oder eine Wertpapierfirma“, und Art. 2 Abs. 1 Nr. 2 RL 2014/59/EU definiert ein Kreditinstitut mit Verweis auf Art. 4 Abs. 1 Nr. 1 VO 575/2013.$^{54}$ Diese Bestimmung definiert wiederum ein Kreditinstitut als „ein Unternehmen, dessen Tätigkeit darin besteht, Einlagen oder andere rückzahlbare Gelder des Publikums entgegenzunehmen und Kredite für eigene Rechnung zu gewähren“".

Grundsätzlich kann somit durchaus auf das Bestehen einer Zulassung, d.h. hier einer Bankenkonzession, abgestellt werden, um ein Unternehmen gemäß dem Wortlaut als Kreditinstitut und somit als Institut im Sinne der Richtlinie einzustufen. Zugleich bietet jedoch der Wortlaut bzw. die Legaldefinition eines Kreditinstitutes im Sinne der RL 2014/59/EU einen gewissen Spielraum. Weder der Begriff „Kreditinstitut“ noch die Definition in der Verordnung 575/2013 klären eindeutig, in welchem Umfang ein Unternehmen Bankengeschäfte zulässigerweise vornehmen können muss, um noch als Kreditinstitut zu gelten. Gewiss muss es dazu in der Lage sein, da Art. 4 Abs. 2 Nr. 1 VO 575/2013 davon spricht, dass ,dessen Tätigkeit“ darin bestehen muss.

\footnotetext{
$51 \quad$ Hierzu noch näher B.III.

52 Hierzu noch näher unten $\mathrm{C}$.

53 Für den vorliegenden Sachverhalt ist nur diese Bestimmung von Belang, so dass sich eine Erörterung der anderen Ziffern des Art. 1 Abs. 1 RL 2014/59/EU, die etwa die Fälle von Tochterunternehmen oder Finanzholdinggesellschaften regeln, nicht aufdrängt. 
Fraglich - und im vorliegenden Zusammenhang relevant bzw. entscheidend - ist jedoch, ob diese Tätigkeit in jedem Fall einen wesentlichen Inhalt der Geschäfte eines Kreditinstituts darstellen muss. Grundsätzlich legt der Wortlaut der Bestimmung die Bejahung dieser Frage nahe, da in diesem Tätigkeitsbegriff gemäß der Definition von Art. 4 Abs. 1 Nr. 1 VO 575/2013 „mitschwingt“, dass es sich um einen Teil der hauptsächlichen Geschäftstätigkeit handeln muss. Vor diesem Hintergrund wäre es aufgrund des Wortlauts a priori denkbar, eine streng statische Betrachtungsweise auf den Begriff des Institutes anzuwenden. ${ }^{55}$ Gemäß einer solchen Lesart ist bzw. wäre auch eine Abbaueinheit in jedem Fall ab dem Zeitpunkt des Wegfallens der Zulassung von der späteren Anwendung der RL 2014/59/EU ausgeschlossen. Allerdings eröffnet der Wortlaut aber auch eine andere Lesart (wird doch allgemein auf die „Tätigkeit“ abgestellt) und schließt es nicht von vornherein aus, dass zumindest in gewissen Konstellationen eine insgesamt untergeordnete „Bankentätigkeit“ eines Instituts für dessen Qualifizierung als Kreditinstitut ausreicht. In Betracht kommt dies insbesondere für eine „Restzulassung“ für eine „Bankentätigkeit“, die den Abwicklungszielen der Abbaueinheit untergeordnet ist bzw. nur diesen beschränkten Zielsetzungen dient, dies jedenfalls soweit das Institut vorher als Kreditinstitut tätig war. Soweit die Situation der HETA betroffen ist, wurde diese zwar zum Zeitpunkt des Ablaufes der Umsetzungsfrist der RL 2014/59/EU bereits als Abbaueinheit geführt. Jedoch kann sie auch nach dem Wegfall der (ursprünglich gegebenen) Konzession zum Betrieb von Bankgeschäften immer noch aufgrund einer gesetzlichen Konzession Bank- und Leasinggeschäfte betreiben sowie Beteiligungsankäufe und -verkäufe vornehmen, sofern dies unmittelbar oder mittelbar der Aufgabenerfüllung dient. ${ }^{56}$ Auf dieser Grundlage erscheint es durchaus vertretbar, dass die HETA trotz ihrer Qualifizierung als Abbaueinheit noch unter den Begriff eines Kreditinstitutes fällt. Sie ist zwar keine werbende Bank mehr, aber immerhin noch eine in Abwicklung befindliche Einheit, die in begrenztem Umfang weiterhin Bankgeschäfte betreiben kann .

Für diesen Ansatz sprechen auch der Regelungsgegenstand der RL 2014/59/EU und der Wortlaut anderer Bestimmungen: Insgesamt zielt die Richtlinie auf die Errichtung eines Abwicklungsregimes, so dass es wenig überzeugend erscheint, aufgrund einer streng statischen Position davon auszugehen, dass ein Institut mit dem Entzug der Zulassung notwendigerweise - und unabhängig vom genauen Anlass des Entzuges - in ein anderes Regime, nämlich jenes des „normalen“ Insolvenzrechtes, eintreten müsse. Überzeugender erscheint hier vielmehr eine „dynamische“ Perspektive, die zu berücksichtigen vermag, dass ein Institut zu einem früheren Zeitpunkt die Qualität eines Institutes mit umfassender Zulassung, hier einer Bankenkonzession, aufwies und somit in den Anwendungsbereich der RL 2014/59/EU gefallen wäre und zugleich auch nicht aus diesem herausfällt, wenn im Rahmen eines Abwicklungsprozesses, auch wenn dessen erste Phasen sich vor Ablauf der Umsetzungsfrist der Richtlinie abspielen, die Zulassung entzogen wird. Dem widerspricht auch nicht der Wortlaut der RL 2014/59/EU, der „Vorschriften und Verfahren für die Sanierung und Abwicklung“ von Unternehmen festlegt, jedoch dabei nicht festlegt, ob die Vorgaben nicht auch auf Prozesse zur Anwendung kommen können, die bereits vor Ablauf der Umsetzungsfrist eingeleitet worden sind. ${ }^{57}$

Damit bleibt festzuhalten, dass es der Wortlaut der relevanten unionsrechtlichen Bestimmungen durchaus erlauben würde, ein Institut in der Situation der HETA in den Anwendungsbereich der RL 2014/59/EU einzubeziehen.

$55 \quad$ Etwa bei Fest (Fußn. 7), S. 1110.

$56 \quad \S 3$ Abs. 4 GSA.

57 Art. 1 Abs. 1 S. 1 RL 2014/59/EU. 


\section{b) Teleologische Auslegung}

Für einen somit durch den Wortlaut der relevanten unionsrechtlichen Bestimmungen ermöglichten zwingenden Einbezug eines Instituts in der Situation der HETA und damit ein dynamisches Verständnis des persönlichen Anwendungsbereichs der RL 2014/59/EU sprechen sodann und vor allem teleologische Gesichtspunkte. Als Zielsetzung ist in RL 2014/59/EU die Schaffung eines glaubwürdigen Sanierungs- und Abwicklungsrahmens für den wirksamen Umgang mit unsoliden oder ausfallenden Kreditinstituten und Wertpapierfirmen festgehalten. ${ }^{58}$ Dafür wird ein kohärenter Regelungsrahmen benötigt, und es muss das höchstmögliche Niveau an Finanzstabilität für alle Institute sichergestellt werden. ${ }^{59}$ Diese Zielsetzungen könnten durch eine statisch orientierte Betrachtungsweise, die primär auf den Bestand einer Zulassung abstellt und ab diesem Zeitpunkt die Einleitung eines „normalen“ Insolvenzverfahrens zur Folge hätte, kaum erreicht werden. Auch ist kein schützenswertes Interesse erkennbar, das ein solches Vorgehen begründen könnte. Weder ist ersichtlich, warum etwa sich in einem Abwicklungsverfahren befindliche Unternehmen schutzwürdige, legitime Interessen haben sollten, von der Anwendung einer späteren Rechtsentwicklung in Form der RL 2014/59/EU ausgenommen zu werden, noch gilt dies für Behörden anderer Mitgliedstaaten, auch wenn sich für sie Rechtswirkungen etwa aus dem dann anwendbaren Art. 3 Abs. 2 RL 2001/24/EG ergeben; ${ }^{60}$ gerade bei solchen Behörden ist kein Grund ersichtlich, warum sie etwa zur Nichtanerkennung von nach Ablauf der Umsetzungsfrist ergriffenen Abwicklungsmaßnahmen im Rahmen des neu anwendbaren unionsrechtlichen Abwicklungsregimes berechtigt sein sollten, dies nur weil erste Abwicklungsschritte bereits vor Ablauf der Umsetzungsfrist ergriffen worden sind. Insbesondere ist festzuhalten, dass es hier gerade nicht um eine rückwirkende Anwendung der Richtlinie, sondern um die Präzisierung des Anwendungsbereichs der Richtlinie geht, der eben solche früher eingeleiteten Prozesse auch umfasst, woraus sich Rechtswirkungen für nach Ablauf der Umsetzungsfrist ergriffene Abwicklungsmaßnahmen ergeben.

Darüber hinaus ist das primäre Ziel des durch die RL 2014/59/EU geschaffenen Abwicklungsregimes nicht die Bewahrung oder Stabilisierung einer Institution, sondern deren Transformation in eine Einheit, von der keine Bedrohung für den Finanzmarkt bzw. Bedarf einer Stützung durch staatliche Mittel ausgeht. Im Visier der RL 2014/59/EU stehen, wie sich anhand der Voraussetzungen für die Anwendung von Abwicklungsmaßnahmen zeigt, Institute, die bereits in einem Zustand sind oder demnächst sein werden, in dem ihnen keine Bankenkonzession mehr gewährt würde. ${ }^{61}$ Somit erscheint eine Auslegung widersinnig, die die Anwendung der Richtlinie auf ein Institut verneint, nur weil es wegen des Vorliegens derselben Voraussetzungen bereits über keine Bankenkonzession mehr verfügt. Besonders betont wird an dieser Stelle bereits in der Richtlinie selbst, dass das Abwicklungsregime ein „rechtzeitiges“ Eintreten in die Abwicklung vorsehen soll, ${ }^{62}$ was eben genau ein Vorgehen wie jenes des österreichischen Gesetzgebers in der Form des GSA bzw. des BaSAG erfassen dürfte.

Der Zielsetzung der RL 2014/59/EU liefe es daher wohl auch zuwider, sie in einem Fall nicht zur Anwendung zu bringen, in dem wegen der Anwendung eines Abwicklungsinstrumentes vor Ablauf der Umsetzungsfrist der Richtlinie eine Einheit wie die HETA keine Zulassung mehr hat und daher bei Ablauf der Umsetzungsfrist bei statischer Betrachtung nicht als

\footnotetext{
$58 \quad$ Siehe Erw. 1 RL 2014/59/EU.

59 Erw. 11 RL 2014/59/EU.

60 Hierzu oben B.II.1.

$61 \quad$ Art. 32 Abs. 4 Ziff. a RL 2014/59/EU.

62 Erw. 41 RL 2014/59/EU.
} 
Institut eingestuft werden könnte. ${ }^{63}$ Dies schlösse nämlich gerade schwere Fälle wie jenen der Schaffung der HETA als Abbaueinheit vom neuen unionsrechtlichen Abwicklungsregime aus.

Eine Position, die streng auf das Bestehen einer Zulassung abstellt, brächte zudem die Gefahr eines widersprüchlichen Verhaltens der mitgliedstaatlichen Behörden mit sich. Die Zielsetzungen der Richtlinie betonen nämlich, dass eine qualifizierte Verletzung der Zulassungsvoraussetzungen als Institut erforderlich ist, damit ein Abwicklungsverfahren eingeleitet werden kann. ${ }^{64}$ Somit müsste einerseits für die Anwendung der Richtlinie festgestellt werden, dass in qualifizierter Weise die Voraussetzungen für eine Gewährung einer Zulassung nicht vorlägen, während aufsichtsrechtlich jedoch, um die Richtlinie anzuwenden, eben die Zulassung nicht entzogen werden dürfte. Auch wird gerade durch die Anwendung der Abwicklungsinstrumente eine „Entkernung“ eines Institutes vorgenommen; insofern bleibt fraglich, weshalb hierbei darauf abgestellt werden sollte, dass das Institut rein formell seine Zulassung behält und weiter werbendes Geschäft betreiben kann, ganz abgesehen davon, dass die werbenden Geschäfte eines solchen Institutes die Erreichung der Ziele des Abwicklungsregimes stören könnten.

Insgesamt widerspricht es so der Zielsetzung des von der RL 2014/59/EU errichteten Abwicklungsregimes, wenn für den Anwendungsbereich der Richtlinie streng darauf abgestellt wird, dass ein Institut eine Zulassung besitzt. Ein strenges Abstellen auf die Zulassung führte dazu, dass bei einem Institut bei Verlust der Zulassung auch in einem Abwicklungsprozess plötzlich das ,normale“ Insolvenzrecht bzw. -verfahren zur Anwendung käme, was klar den Zielsetzungen des eben speziell für den Fall derartiger Institute geschaffenen Abwicklungsregimes zuwider liefe.

\section{c) Systematische Auslegung}

Eine prozessbezogene und daher weite Auslegung des Begriffs des Institutes lässt sich auch im Rahmen einer systematischen Auslegung durch die Bezugnahme auf andere Bestimmungen begründen. So werden zwar als Teil der Definition von Abwicklungsmaßnahmen in der RL 2014/59/EU Entscheidungen über die Abwicklung eines Institutes angeführt, die wohl typischerweise ein Institut, d.h. eine Bank mit Bankenkonzession, betreffen. Andererseits jedoch umfasst der Begriff auch die „Anwendung eines Abwicklungsinstruments“ oder die „Ausübung einer oder mehrere Abwicklungsbefugnisse“. ${ }^{65}$ Beide Maßnahmen können sich auch auf eine Einheit beziehen, die nach strenger Auffassung bereits nicht länger ein Institut darstellt, etwa eine Abbaueinheit nach Verlust der Zulassung. Die RL 2014/59/EU stellt somit nicht streng auf den Zeitpunkt des Verlustes der Zulassung ab, sondern betrachtet die Abwicklung als Prozess.

Eine ähnliche Schlussfolgerung ergibt sich aus der Betrachtung der Voraussetzungen für die Anwendung einer Abwicklungsmaßnahme. Die RL 2014/59/EU sieht vor, dass unter anderem Abwicklungsmaßnahmen nur dann zu treffen sind, wenn die zuständige Behörde nach Anhörung der Abwicklungsbehörde oder gegebenenfalls die Abwicklungsbehörde der Ansicht ist, dass ein Institut ausfällt oder wahrscheinlich ausfällt. ${ }^{66}$ Letzteres ist dann anzunehmen, wenn ein Institut gegen Anforderungen einer dauerhaften Zulassung derart verstösst, dass der Verlust der Zulassung gerechtfertigt wäre bzw. dies in naher Zukunft der

$63 \quad$ Perner (Fußn. 5), S. 242.

64 Erw. 41 RL 2014/59/EU.

65 Art. 2 Abs. 1 Ziff. 40 RL 2014/59/EU.

66 Art. 32 Abs. 1 Bst. a RL 2014/59/EU. 
Fall sein könnte ${ }^{67}$ Hieraus ergibt sich jedoch lediglich, dass zum Zeitpunkt der Prüfung der Voraussetzungen für den Einsatz von Abwicklungsmaßnahmen ein Institut über eine Zulassung verfügen muss; im Folgenden muss aber dies aufgrund der Richtlinie nicht für jede Anwendung einer Abwicklungsmaßnahme erneut festgestellt werden, d.h. auch nicht unbedingt stets eine Zulassung für das betroffene Institut vorliegen.

Denselben prozessorientierten Gedanken bringt die Richtlinie in Bezug auf die durch Abwicklungsbehörden zu berücksichtigenden Elemente zum Ausdruck. So sollen die Behörden nicht nur bei der „Festlegung und Anwendung der Anforderungen“ der Richtlinie, sondern auch bei der „Anwendung“ der Abwicklungsinstrumente unter Berücksichtigung gewisser Charakteristika des betroffenen Unternehmens vorgehen. ${ }^{68}$ Auch dies spricht für eine prozessorientierte Lesart des Anwendungsbereiches der RL 2014/59/EU.

Als weiteres Argument, das eine weite Auslegung des Anwendungsbereichs der RL 2014/59/EU zu begründen vermag, kann Art. 37 Abs. 5 RL 2014/59/EU herangezogen werden. Nach dieser Bestimmung dürfen die Abwicklungsbehörden das Abwicklungsinstrument der Ausgliederung von Vermögenswerten nur zusammen mit einem anderen Abwicklungsinstrument anwenden. Bei einer engen Auslegung des Begriffs des Instituts fiele nunmehr der Fall, in dem ein Abwicklungsinstrument bereits vor Ablauf der Umsetzungsfrist angewendet wurde, aus dem Anwendungsbereich der Richtlinie heraus. Auf diese Art würden nunmehr sämtliche anderen Abwicklungsinstrumente der Richtlinie ausgehebelt. Gerade vor dem Hintergrund, dass Art. 37 Abs. 5 RL 2014/59/EU eindeutig zeigt, dass die Richtlinie ein ganzes Abwicklungsregime schaffen will, dessen einzelne Elemente miteinander zusammenhängen, überzeugt es wenig zuzulassen, dass einzelne Teile des Regimes auf diese Art herausgelöst werden könnten. ${ }^{69}$

Die Richtlinie in einem solchen Fall nicht anzuwenden, hieße überdies, Art. 37 Abs. 5 RL 2014/59/EU seines effet utile zu berauben, da seine Funktion als verpflichtendes Bindeglied zwischen dem Abwicklungsinstrument der Ausgliederung von Vermögenswerten und einem oder mehreren anderen Abwicklungsinstrumenten ausgehöhlt würde.

Abgesehen von anderen Richtlinienbestimmungen kann im weiteren Zusammenhang auch auf andere Rechtsakte verwiesen werden. Insbesondere fällt auf, dass kein unmittelbarer Zusammenhang zwischen den unionsrechtlichen Regelungen bezüglich der Abwicklung und den Vorgaben zur Erteilung einer Zulassung besteht. Die RL 2014/59/EU schafft zu diesem Zweck keine Verbindung, etwa über einen eindeutigen Verweis oder ähnliches, zum aufsichtsrechtlichen Regime der Verordnung 575/2013. ${ }^{70}$

Im Vergleich dazu ist etwa in der RL 2001/24/EG ausdrücklich geregelt, dass bei Eröffnung eines Liquidationsverfahrens, wenn keine Sanierungsmaßnahmen getroffen wurden oder diese gescheitert sind, auch die Zulassung des betroffenen Kreditinstitutes zu widerrufen ist. ${ }^{71}$ Begründet wird dies damit, dass ein solcher Widerruf eine notwendige Folge der Liquidation sei und nur noch in begrenztem und kontrolliertem Umfang eine Weiterführung der Tätigkeit möglich sein solle. ${ }^{72}$ Während also im Fall eines Liquidationsverfahrens ausdrücklich der Verlust der Zulassung als Bedingung vorgesehen ist, drängt sich der Umkehrschluss auf, dass das Fehlen einer solchen eindeutigen Regelung in der RL 2014/59/EU eben darauf hinweist, dass nicht streng auf das Vorliegen einer Zulassung abzustellen ist, damit der Anwendungsbereich der RL 2014/59/EU eröffnet ist.

\footnotetext{
$67 \quad$ Art. 32 Abs. 4 Bst. a RL 2014/59/EU.

68 Art. 1 Abs. 1 S. 2 RL 2014/59/EU.

$69 \quad$ Perner (Fußn. 5), S. 242.

70 ABl. $2013 \mathrm{~L} 176,1 \mathrm{ff}$.

71 Art. 12 Abs. 1 RL 2001/24/EG.

72 Erw. 19 RL 2001/24/EG.
} 


\section{d) Folgenabschätzung einer engen Auslegung des Anwendungsbereichs und Sperrwirkung der RL 2014/59/EU}

Fraglich scheint zuletzt, ob es einem nationalen Gesetzgeber gestattet sein sollte, durch ein zeitlich der Richtlinie vorgreifendes Vorgehen ein Kreditinstitut durch vorzeitige Anwendung eines Abwicklungsinstrumentes dem in der Richtlinie vorgesehenen Abwicklungsregime zu entziehen. Die Absicht des österreichischen Gesetzgebers war im vorliegenden Sachverhalt zwar das Gegenteil, nämlich die Schaffung einer Abbaueinheit im Einklang mit den unionsrechtlichen Vorgaben der bereits im Amtsblatt veröffentlichten RL 2014/59/EU durch den Erlass des GSA bzw. durch die nach Ablauf der Umsetzungsfrist der Richtlinie erfolgte Einbeziehung der HETA in das allgemeine, durch das BaSAG geschaffene Bankenabwicklungsregime in $\S 162$ Abs. 6 BaSAG.

Jedoch bestünde diese Möglichkeit unabhängig vom vorliegenden Fall, wenn man einer engen Auslegung des Anwendungsbereichs der RL 2014/59/EU folgt. Durch sein noch nicht durch die Richtlinie eingeschränktes Handeln hätte der Gesetzgeber so theoretisch absichtlich eine Abwicklung unter Entzug der Zulassung vor Ablauf der Umsetzungsfrist der Richtlinie einleiten können, um die Anwendung der Richtlinie auf die späteren Phasen des Abwicklungsprozesses zu vermeiden.

Eine solche Folgenabschätzung stellt zwar streng genommen keine Auslegungsmethode dar. Hingegen lässt sie sich dogmatisch auf die - begrenzte ${ }^{73}$ - Sperrwirkung der Richtlinie abstützen. Aufgrund dieser Sperrwirkung ist es nämlich untersagt, Maßnahmen zu ergreifen, die das Richtlinienziel ernstlich in Frage stellen können. ${ }^{74}$ Auf die subjektive Absicht des Gesetzgebers kommt es bei den ergriffenen Maßnahmen nicht an. ${ }^{75}$ Zeitlich kann davon ausgegangen werden, dass die Vorwirkung der RL 2014/59/EU bereits mit der Veröffentlichung im Amtsblatt im Juni 2014 und somit vor Erlass des GSA bzw. der Schaffung der Abbaueinheit einsetzte. Das Frustrationsverbot ${ }^{76}$ könnte hier verletzt sein, wenn die Schaffung der Abbaueinheit nach der einen engen Anwendungsbereich der Richtlinie vertretenden Auffassung dazu führt, dass das Richtlinienziel nicht mehr erreicht werden kann.

Ziel der Richtlinie ist die Schaffung eines glaubwürdigen Sanierungs- und Abwicklungsrahmens, der den zuvor bestehenden Mangel an angemessenen Instrumenten für den wirksamen Umgang mit unsoliden oder ausfallenden Kreditinstituten beheben soll, da sich die Anwendung der allgemeinen, nicht harmonisierten Insolvenzverfahren in den Mitgliedstaaten auf derartige Fälle nicht eignet. ${ }^{77}$ Bereits die Erreichung dieser Zielsetzung wäre gefährdet, wenn man die Schaffung einer Abbaueinheit, auf die trotz der potentiellen Gefahr, die vom betroffenen Institut ausgeht, nicht die übrigen Vorgaben der RL 2014/59/EU zur Anwendung kommen, akzeptiert.

Darüber hinaus soll die Richtlinie auch eine Gleichbehandlung der Kreditinstitute, was den Zugang zu Liquidität und ähnliche Maßnahmen betrifft, sicherstellen. ${ }^{78}$ Auch vor diesem Hintergrund überzeugt eine Sonderbehandlung der HETA als Abbaueinheit, auf die die RL 2014/59/EU keine Anwendung findet, nicht.

\footnotetext{
73 Wie zuvor bereits angesprochen, siehe oben B.I.2.

74 EuGH, Rs. C-129/96 (Inter-Environnement Wallonie), EU:C:1997:628, Rdnr. 45.

$75 \quad$ Perner (Fußn. 5), S. 243.

$76 \quad$ Kühling (Fußn. 42), S. 858.

77 Erw. 1 und 4 RL 2014/59/EU.

78 Erw. 2 RL 2014/59/EU.
} 
Alle Ziele der Richtlinie sollen dadurch erreicht werden, dass den Behörden der Mitgliedstaaten in der Richtlinie ein ,zuverlässiges Instrumentarium“ an die Hand gegeben wird, um rechtzeitig und rasch intervenieren zu können. ${ }^{79}$ Dieses ,,übergreifende“ Ziel wäre gefährdet, nähme man eine Nichtanwendbarkeit der RL 2014/59/EU in einer Konstellation wie der vorliegenden an. In einem solchen Fall stünde den Behörden überhaupt kein Instrumentarium für den Fall der HETA zur Verfügung, da sämtliche Abwicklungsinstrumente der Richtlinie somit ausgeschlossen gewesen wären. Hinzu kommt, dass auch der bewusst in der Richtlinie geschaffene Zusammenhang zwischen den Abwicklungsinstrumenten nicht zur Anwendung käme. Art. 37 Abs. 5 RL 2014/59/EU sieht nämlich vor, dass auf das Instrument der Ausgliederung von Vermögenswerten nicht allein, sondern nur zusammen mit einem anderen Instrument zurückgegriffen werden soll. Auch dieser Aspekt, der wohl als Teil des Konzeptes eines „zuverlässigen Instrumentariums“ verstanden werden muss, bliebe somit bei einer ,statischen“ Sichtweise unberücksichtigt.

Darüber hinaus soll durch die RL 2014/59/EU sichergestellt werden, dass Verluste primär von den Anteilseignern und erst danach von den Gläubigern getragen werden. ${ }^{80}$ Auch diesem würde durch ein einzelfallbezogenes Vorgehen im Fall der HETA ohne Anwendung der einschlägigen Vorgaben der RL 2014/59/EU nicht Rechnung getragen.

Schließlich ist zu betonen, dass die vorstehenden Erwägungen keine Verpflichtung des österreichischen Gesetzgebers implizieren, die gesamte RL 2014/59/EU vorzeitig umzusetzen. Sie bewirkt lediglich, dass er, wenn er sich entscheidet, eine Bankenabwicklung wie im Fall der HETA in der Phase zwischen Veröffentlichung der RL 2014/59/EU im Amtsblatt und Ablaufen der Umsetzungsfrist der Richtlinie einzuleiten, eine „stufenweise“ Umsetzung zu wählen hat, bei der die Abbaueinheit, wie im vorliegenden Fall auch geschehen, ab Ablauf der Umsetzungsfrist trotz möglichem Entzug der Zulassung als Institut gemäß den Verpflichtungen aus der Richtlinie behandelt wird. ${ }^{81}$

Daher überzeugt insgesamt auch auf der Grundlage einer Folgenabschätzung die Annahme nicht, die RL 2014/59/EU komme nicht auf den Fall einer vor Ablauf der Umsetzungsfrist der Richtlinie geschaffenen Abbaueinheit zur Anwendung. Vielmehr führte - im Gegenteil die Verneinung der Eröffnung des persönlichen Anwendungsbereichs der Richtlinie wohl zu einer Verletzung des Frustrationsverbotes. In Bezug auf den österreichischen Gesetzgeber ist dabei nochmals $\mathrm{zu}$ betonen, dass dieser bewusst die Anwendung der unionsrechtlichen Verpflichtungen aus der Richtlinie auf den geradezu typischen Anwendungsfall der Schaffung der HETA als Abbaueinheit vorziehen wollte.

\section{e) Zusätzliche Fragen des Anwendungsbereichs der RL 2014/59/EU}

Weiter könnte sich die Frage stellen, ob der Fall der Schaffung einer Abbaueinheit wie der HETA in den Anwendungsbereich der RL 2014/59/EU fällt, da es sich um ein Unternehmen ohne Zweigstellen bzw. um ein verstaatlichtes Institut handelt.

Dafür, dass die Richtlinie auch auf Unternehmen ohne Zweigstellen Anwendung finden kann, spricht bereits Art. 1 Abs. 1 der Richtlinie, der an keiner Stelle formuliert, dass ein Unternehmen Zweigstellen haben müsse, sondern etwa von Instituten, „die in der Union niedergelassen sind“, spricht. Auch in der Zielsetzung ist von ,grenzüberschreitend tätigen“ Instituten die Rede, die die Stabilität der Finanzmärkte in einzelnen Mitgliedstaaten, in denen

\begin{tabular}{ll}
\hline 79 & Erw. 5 RL 2014/59/EU. \\
80 & Erw. 5 RL 2014/59/EU. \\
81 & So überzeugend Perner (Fußn. 5), S. 244.
\end{tabular}


sie ,tätig“ seien, beeinflussen könnten. ${ }^{82}$ Erneut wird auf die potentiellen Auswirkungen der Tätigkeit eines Institutes und nicht auf das Bestehen von Zweigstellen in anderen Mitgliedstaaten abgestellt.

Der Richtlinie lässt sich darüber hinaus kein Hinweis entnehmen, dass die Verstaatlichung eines Institutes etwas an der Anwendbarkeit der Richtlinie ändert. Sowohl beim Gegenstand und Anwendungsbereich ${ }^{83}$ als auch bei den Zielsetzungen ${ }^{84}$ werden lediglich Institute genannt, ohne dass auf deren konkrete Eigentumssituation näher eingegangen würde. Somit fallen sowohl Institute ohne Zweigstellen in anderen Mitgliedstaaten als auch verstaatlichte Institute in den Anwendungsbereich der RL 2014/59/EU.

\section{Zwischenergebnis}

Im Ergebnis ist somit - im Anschluss an die Auslegung der RL 2014/59/EU auf der Grundlage der anerkannten Auslegungsmethoden - festzuhalten, dass nur eine Auslegung, die den Prozesscharakter von Abwicklungsvorgängen angemessen berücksichtigt, zu überzeugen vermag. Eine statische Perspektive, die für den Begriff des Institutes, das in den Anwendungsbereich der RL 2014/59/EU fällt, nur auf den Zeitpunkt des Wegfalls der Zulassung abstellt, wird dem nicht gerecht. Daraus ergibt sich eine Verpflichtung für den mitgliedstaatlichen Gesetzgeber, bei der Umsetzung der Richtlinie in das nationale Recht sicherzustellen, dass auch Unternehmen rechtlich erfasst werden, bei denen bereits erste Schritte eines Abwicklungsprozesses, die auch den Verlust der Zulassung umfassen können, erfolgt sind und deren Situation zugleich eine typische Gefahrenlage schafft, auf deren Bewältigung die Richtlinie abzielt. Da dies im Fall der HETA zu bejahen sein dürfte, hat der österreichische Gesetzgeber die RL 2014/59/EU somit unionsrechtskonform umgesetzt, indem er durch das GSA die Schaffung einer Abbaueinheit unter bereits der Richtlinie entsprechenden Vorgaben sicherstellte und ab Ablaufen der Umsetzungsfrist der Richtlinie im BaSAG die Anwendung der Richtlinienvorgaben auf die HETA erneut ausdrücklich und insofern auch rückwirkend sicherstellte. Festzuhalten ist allerdings auch, dass die volle Rechtswirkung der RL 2014/59/EU erst mit 1.1.2015 einsetzt und somit auch konkret getroffene Abwicklungsmaßnahmen erst ab diesem Datum der Richtlinie unterliegen bzw. auch dementsprechend unionsrechtlich determinierte Rechtswirkungen entfalten. ${ }^{85}$

\section{Zum sachlichen Anwendungsbereich der RL 2014/59/EU und der RL 2001/24/EG}

Nachdem die Frage des persönlichen Anwendungsbereiches der RL 2014/59/EU in Bezug auf Institute, die ihre Zulassung im Rahmen von Abwicklungsprozessen bereits vor Ablauf der Umsetzungsfrist der Richtlinie verlieren, geklärt wurde, ist an dieser Stelle näher auf den sachlichen Anwendungsbereich der RL 2014/59/EU und 2001/24/EG einzugehen. So ist zu untersuchen, inwieweit das GSA als Abwicklungsmaßnahme einzustufen ist und somit in den sachlichen Anwendungsbereich der RL 2014/59/EU fällt.

Alternativ dazu könnte das GSA auch eine Sanierungsmaßnahme oder Teil eines Liquidationsverfahrens im Sinne der RL 2001/24/EG bzw. der vor Ablauf der

$82 \quad$ Erw. 3 RL 2014/59/EU.

83 Art. 1 RL 2014/59/EU.

$84 \quad$ Etwa Erw. 3, 4 und 5 RL 2014/59/EU.

$85 \quad \mathrm{Zu}$ den Rechtswirkungen des Moratoriums der FMA noch unten C. 
Umsetzungsfrist der RL 2014/59/EU bestehenden Rechtslage darstellen. Für die Rechtsfolgen einer solchen Einstufung kann in beiden Fällen bereits auf vorherige Ausführungen verwiesen werden. ${ }^{86}$

\section{Zum GSA als Abwicklungsmaßnahme und Vorwegnahme der Umsetzung der RL 2014/59/EU}

Das GSA ist dann als eine Abwicklungsmaßnahme im Sinne der RL 2014/59/EU und damit in Bezug auf die Schaffung der HETA eine Maßnahme zur (vorweggenommenen) Umsetzung der Vorgaben der RL 2014/59/EU anzusehen, wenn es tatsächlich die einschlägigen Vorgaben der Richtlinie umsetzt bzw. umsetzen soll, so dass im Folgenden zuerst die einzelnen (hier relevanten) Vorgaben der Richtlinie skizziert und auf dieser Grundlage in Bezug zum GSA gesetzt werden.

Ein Abwicklungsverfahren soll nach den Voraussetzungen von Art. 32 und 33 der RL 2014/59/EU dann zur Anwendung kommen, wenn der Ausfall eines Kreditinstituts innerhalb eines angemessenen Zeitrahmens wahrscheinlich nicht abgewendet werden kann und wenn ein öffentliches Interesse an der Abwicklung besteht. ${ }^{87}$ Für das Bestehen eines öffentlichen Interesses muss eine Abwicklungsmaßnahme für das Erreichen mindestens eines Abwicklungsziels ${ }^{88}$ der Richtlinie erforderlich und in Bezug auf dieses Ziel verhältnismäßig sein, während dies bei einer Liquidation auf dem Wege eines regulären Insolvenzverfahrens nicht in demselben Umfang der Fall wäre. ${ }^{89}$

Im Fall der Schaffung der HETA ist nicht ersichtlich, dass das Vorliegen dieser Voraussetzungen bestritten wird bzw. werden soll. Insbesondere dürfte zu bejahen sein, dass etwa das Abwicklungsziel des Schutzes öffentlicher Mittel durch geringere Inanspruchnahme außerordentlicher finanzieller Unterstützung aus öffentlichen Mitteln durch die Schaffung der HETA verfolgt werden soll. ${ }^{90}$ Eine aussagekräftigere Prüfung der Verhältnismäßigkeit und Angemessenheit der getroffenen Maßnahmen aus ökonomischer Sicht kann im vorliegenden Rahmen nicht geleistet werden.

Die in der RL 2014/59/EU vorgesehenen Abwicklungsinstrumente sind - wie bereits dargestellt ${ }^{91}$ - die Unternehmensveräußerungen an Dritte, ${ }^{92}$ Unternehmensveräußerungen an ein Brückeninstitut, ${ }^{93}$ die Ausgliederung von Vermögenswerten auf eine Zweckgesellschaft (sogenannte Bad Bank) ${ }^{94}$ und das Bail-in-Instrument. ${ }^{95}$ Darüber hinaus können die Mitgliedstaaten zusätzliche Instrumente und Befugnisse vorsehen, sofern Gruppenabwicklungen nicht behindert und die Abwicklungsziele und -grundsätze ${ }^{96}$ der Richtlinie beachtet werden. ${ }^{97}$ Somit besteht ein weiter Gestaltungsspielraum für die Mitgliedstaaten, zusätzliche Abwicklungsinstrumente $\mathrm{zu}$ schaffen. Eine vollständige Harmonisierung wird durch die Richtlinie somit nicht angestrebt. Für die folgende Prüfung

\footnotetext{
$86 \quad$ Siehe oben B.II.1.

87 Hierzu etwa Jung/Bischof (Fußn. 13), Rdnr. 163.

88 Art. 31 Abs. 2 RL 2014/59/EU.

89 Art. 32 Abs. 5 RL 2014/59/EU.

$90 \quad$ Art. 31 Abs. 2 Bst. c RL 2014/59/EU.

91 Siehe B.I.1.

$92 \quad$ Art. 38 und 39 RL 2014/59/EU.

93 Art. 40 und 41 RL 2014/59/EU.

94 Art. 42 RL 2014/59/EU.

95 Art. 43, 44 und 48 RL 2014/59/EU.

$96 \quad$ Art. 31 und 34 RL 2014/59/EU.

97 Art. 37 Abs. 9 RL 2014/59/EU.
} 
des GSA bedeutet dies, dass nicht sämtliche getroffenen Einzelmaßnahmen unter die Bestimmungen der Richtlinie subsumierbar sein müssen; um es als Teil eines Abwicklungsverfahrens im Sinne der RL 2014/59/EU einzustufen, ist dennoch als Anforderung zu stellen, dass sein Schwerpunkt im Bereich eines oder mehrerer der Abwicklungsinstrumente der Richtlinie liegt.

Untersucht man in diesem Licht das GSA, ${ }^{98}$ lässt sich eine Unterscheidung zwischen Bestimmungen treffen, die in den Bereich der Richtlinie fallen, und anderen Bestimmungen. Nicht unter die Richtlinie fällt die Ermächtigung des Bundesministers der Finanzen, eine Aktiengesellschaft, die „ABBAG - Abbaubeteiligungsaktiengesellschaft des Bundes“, zu errichten und die Anteile an der Hypo Alpe-Adria-Bank International AG bzw. der Abbaueinheit und die damit zusammenhängenden Forderungen und Verbindlichkeiten in besagte Aktiengesellschaft einzubringen. ${ }^{99}$ Eine Anteilsveräußerung bzw. -übertragung erfolgt hier nämlich nicht im Rahmen einer abwicklungsrechtlichen Festlegung durch den Minister als Behörde, sondern rechtsgeschäftlich.

Hingegen ist es im Rahmen der Richtlinie als Abwicklungsmaßnahme einzuordnen, wenn das GSA den Bundesminister für Finanzen ermächtigt, durch Verordnung zur Schaffung einer Abbaueinheit Teile der Hypo Alpe-Adria-Bank International AG auf den Bund oder einen anderen aufnehmenden Rechtsträger gegen angemessenes Entgelt auszugliedern, ${ }^{100}$ auch wenn der Erwerb von Vermögenswerten gegebenenfalls auch rechtsgeschäftlich erfolgen kann. ${ }^{101}$ Das GSA bestimmt hier überdies, dass, soweit Gläubigern der Hypo Alpe-AdriaBank International AG Kündigungs-, Zustimmungs- oder andere Gestaltungsrechte oder Rechte auf Sicherstellung ihrer Forderungen zukommen, diese im Fall einer solchen Übertragungsanordnung oder auch einer rechtsgeschäftlichen Übertragung „nicht anzuwenden und nicht ausübbar" seien. ${ }^{102}$

Tatsächlich veräußerte die Hypo Alpe-Adria-Bank International AG Anteile an anderen Instituten; das Unternehmen selbst wurde umgewandelt, indem es sein Einlagengeschäft einstellte, die Firma auf HETA geändert wurde und seine Aufgaben abgeändert wurden, um den Abwicklungszielen des GSA gerecht zu werden. ${ }^{103}$ An dieser Stelle ist - soweit ohne vertiefte ökonomische Untersuchung machbar - dieses Vorgehen im Licht der Abwicklungsinstrumente der RL 2014/59/EU zu prüfen.

Im Sinne der Richtlinie kann das GSA bzw. die tatsächlichen Vorgänge rund um die Schaffung der HETA als eine Anwendung der Abwicklungsinstrumente der Unternehmensveräußerung ${ }^{104}$ und der Ausgliederung von Vermögenswerten eingestuft werden. Bei der Unternehmensveräußerung werden einem Erwerber, der nicht ein Brückeninstitut sein darf, von einem in Abwicklung befindlichen Institut ausgegebene Anteile, Vermögenswerte, Rechte und Verpflichtungen gesamthaft oder teilweise übertragen. ${ }^{105}$ Dies lässt sich wohl für die Veräußerung von Anteilen an andere Institute bejahen, da es sich bei den Erwerbern um keine Zweckgesellschaften, sondern etwa die FIMBAG Finanzmarktbeteiligung AG des Bundes bzw. die HBI-Bundesholding AG handelt. Abwicklungsbehörde ist, wie auch die Richtlinie es zulässt, der zuständige Minister. ${ }^{106}$ Der

98 Nicht einzugehen ist an dieser Stelle auf andere Maßnahmen des österreichischen Gesetzgebers wie etwa das Hypo Alpe-Adria-Bank-Sanierungsgesetz.

$99 \quad \S 2$ Abs. 5 GSA.

$100 \quad \S 1$ Abs. 1 GSA.

$101 \S 1$ Abs. 5 GSA.

$102 \S 1$ Abs. 4 GSA.

103 Siehe im Detail B. einleitender Text.

104 Art. 37 Abs. 3 Bst. a bzw. Art. 38 RL 2014/59/EU.

105 Art. 38 Abs. 1 Bst. a und b RL 2014/59/EU.

106 Art. 3 Abs. 3 RL 2014/59/EU. 
Erwerber muss über die erforderliche Zulassung verfügen, um das erworbene Unternehmen fortführen $\mathrm{zu}$ können. ${ }^{107}$ Hierunter ist aber nicht unbedingt, bzw. nur im Falle einer vollständigen Unternehmensübertragung, eine Bankenkonzession zu verstehen, spricht doch die Bestimmung nur von der „erforderlichen“ Zulassung, ohne etwa zwischen den Fällen einer Übertragung von Anteilen und den Fällen der Übertragung reiner Vermögenswerte zu differenzieren.

Zugleich lässt sich das Vorgehen im GSA auch unter das Instrument der Ausgliederung von Vermögenswerten einordnen. In diesem Rahmen können die zuständigen Behörden Vermögenswerte, Rechte und Verbindlichkeiten eines in Abwicklung befindlichen Institutes auf eine oder mehrere eigens für die Vermögensverwaltung errichtete Zweckgesellschaften übertragen. ${ }^{108}$ Zwar wird im vorliegenden Fall nicht so sehr der „Rumpf“ der Hypo AlpeAdria International AG an eine neu errichtete Zweckgesellschaft übertragen, sondern die Zweckgesellschaft durch die Änderung der Firma in HETA und die Änderung der Unternehmensaufgaben aus dem besagten Rumpf heraus geschaffen. Zumindest faktisch kommt es somit aber zu einer Entkernung der Hypo Alpe-Adria International AG, d.h. es wird eine Maßnahme mit gleicher Wirkung ergriffen. Gemäß Art. 42 Abs. 2 Bst. b RL 2014/59/EU muss eine Zweckgesellschaft „,eigens“ für die Übernahme von Vermögenswerten, Rechten und Verbindlichkeiten eines in Abwicklung befindlichen Institutes „errichtet“ worden sein. Jedoch überzeugt eine rein formalistisch auf die Neuerrichtung einer Zweckgesellschaft abstellende Beurteilung hier nicht. Ob eine für den Abbau der übertragenen Werte der nicht mehr selbständig fortbestehenden Hypo Alpe-Adria International AG bestimmte Zweckgesellschaft neu gegründet wird oder durch Änderung der Firma und der Unternehmensaufgaben aus dem bisherigen Institut hervorgeht, ist wohl kaum die maßgebliche Frage, sofern letzten Endes tatsächlich eine echte Zweckgesellschaft im Sinne der Richtlinie entsteht. ${ }^{109}$ Prüft man die weiteren Voraussetzungen der Ausgliederung von Vermögenswerten, so liegen diese vor. Als Zweckgesellschaft kommt nur eine juristische Person in Frage, die ganz oder teilweise im Eigentum öffentlicher Stellen, wie etwa der Abwicklungsbehörde, steht und von der Abwicklungsbehörde kontrolliert wird und die eigens für die Übernahme bestimmter oder aller Vermögenswerte, Rechte und Verbindlichkeiten des in Abwicklung befindlichen Institutes eingerichtet wurde. ${ }^{110}$ Zwar sind die entsprechenden Vorgaben nicht ausdrücklich so im GSA geregelt, jedoch ist dieses offen genug formuliert, um einer richtlinienkonformen Auslegung zugänglich zu sein. Insbesondere sind die Befugnisse in $\S 1$ Abs. 1 GSA in einem solchen Fall in Übereinstimmung mit den Richtlinienvorgaben auszuüben. Dadurch, dass das Instrument der Ausgliederung von Vermögenswerten nicht isoliert eingesetzt wird, wird das GSA zugleich der entsprechenden Anforderung der RL 2014/59/EU gerecht. ${ }^{111}$

Insgesamt lässt sich somit das GSA als Vorwegnahme der Umsetzung der RL 2014/59/EU (wobei gewisse unionsrechtlich determinierte Rechtswirkungen, insbesondere solche transnationaler Natur, jedoch erst mit Ablauf der Umsetzungsfrist greifen können) und zugleich - ab Ablauf der Umsetzungsfrist der Richtlinie - als Abwicklungsmaßnahme im Sinne der RL 2014/59/EU einstufen. Seine inhaltlichen Vorgaben entsprechen der

\footnotetext{
$107 \quad$ Art. 38 Abs. 7 RL 2014/59/EU.

108 Art. 42 Abs. 1 RL 2014/59/EU.

109 Verneint man dennoch das Vorliegen einer Ausgliederung von Vermögenswerten, könnte es sich beim Vorgang zur Schaffung der HETA um die Anwendung eines - aufgrund Art. 37 Abs. 9 RL 2014/59/EU ausdrücklich zulässigen - Abwicklungsinstruments des nationalen Rechts handeln.

$110 \quad$ Art. 42 Abs. 2 Bst. a und b RL 2014/59/EU.

111 Art. 37 Abs. 5 RL 2014/59/EU.
} 
Anwendung der in der Richtlinie vorgesehenen Abwicklungsinstrumente der Unternehmensveräußerung und der Ausgliederung von Vermögenswerten.

\section{Zum GSA als Sanierungsmaßnahme im Sinne der RL 2001/24/EG}

Bevor das GSA im Rahmen der RL 2001/24/EG daraufhin zu untersuchen ist, ob es eine Sanierungsmaßnahme darstellt (b), müssen die Begriffe der Sanierungsmaßnahme und der Abwicklungsmaßnahme sowie das Verhältnis der sachlichen Anwendungsbereiche der RL 2001/24/EG und der RL 2014/59/EU geklärt werden (a).

Bereits einleitend kann an dieser Stelle eine Einordnung des GSA als Liquidationsverfahren im Sinne der RL 2001/24/EG ausgeschlossen werden. Als solches Verfahren definiert Art. 2 neunter Spiegelstrich der RL 2001/24/EG ,ein von einer Behörde oder einem Gericht eines Mitgliedstaates eröffnetes und unter deren bzw. dessen Aufsicht durchgeführtes Gesamtverfahren mit dem Ziel, die Vermögenswerte unter Aufsicht der genannten Behörden oder Gerichte zu verwerten“. Gerade um ein solches „normales“ Liquidationsverfahren geht es jedoch beim GSA nicht. Wie zuvor bei der Einordnung des GSA im Rahmen der RL 2014/59/EU ausführlich erörtert, geht es hierbei um eine Abwicklung, d.h. eine systematische Veräußerung des betroffenen Unternehmens bzw. der Ausgliederung von Vermögenswerten, um im öffentlichen Interesse Abwicklungsziele wie etwa jenes des Schutzes öffentlicher Mittel zu erreichen. Die „normale“ Verwertung der Vermögenswerte im Liquidationsverfahren ist eben nicht durch das GSA beabsichtigt. Eine Einstufung als Liquidationsverfahren wäre somit widersinnig bzw. trüge den Zielsetzungen des GSA in keiner Weise Rechnung. Für diesen Ansatz spricht auch, dass wohl auch der Unionsgesetzgeber abwicklungsbezogene Maßnahmen wie jene des GSA im Rahmen der RL 2001/24/EG generell eher als den Sanierungsmaßnahmen denn den Liquidationsverfahren verwandt ansah, ordnete er sie doch - als Teil der nun zu diskutierenden Änderungen des Anwendungsbereichs der RL 2001/24/EG durch RL 2014/59/EU - ausdrücklich den Sanierungsmaßnahmen zu, um ihnen die gleichen Rechtswirkungen wie letzteren zukommen zu lassen.

Somit ist in der Folge nur noch zu untersuchen, ob das GSA sich unter den weiter gefassten Begriff der Sanierungsmaßnahmen im Sinne der RL 2001/24/EG fassen lässt.

\section{a) Zum Verhältnis der Anwendungsbereiche der $R L$ 2001/24/EG und der $R L$ 2014/59/EU}

Wie bereits gezeigt, ${ }^{112}$ ändert die RL 2014/59/EU die RL 2001/24/EG in der Form ab, dass die Legaldefinition des Begriffs der „Sanierungsmaßnahmen“ in Art. 2 siebter Spiegelstrich RL 2001/24/EG um den Zusatz ergänzt wird, dass als solche Maßnahmen auch die Anwendung der Abwicklungsinstrumente und die Ausübung der Abwicklungsbefugnisse aus der RL 2014/59/EU zu verstehen sind. ${ }^{113}$

Die genaue Tragweite dieser Modifikation der RL 2001/24/EG erschließt sich nicht auf den ersten Blick. A priori vertretbar erscheinen hier drei Möglichkeiten:

- Erstens kann die Ergänzung der Legaldefinition so gelesen werden, dass der Anwendungsbereich der RL 2001/24/EG erweitert wird, indem neben den bisher mit eigenständiger Bedeutung versehenen Begriff der Sanierungsmaßnahmen jener der

112 Siehe oben B.I.1.

113 Art. 117 Abs. 2 RL 2014/59/EU. 
Abwicklungsmaßnahmen gestellt wird und letztere Maßnahmen zu Sanierungsmaßnahmen ,erklärt" werden.

- $\quad$ Zweitens wäre denkbar, die Ergänzung nur als Konkretisierung zu verstehen, so dass Abwicklungsmaßnahmen bereits bisher - sofern sie die Vorgaben der RL 2001/24/EG für Sanierungsmaßnahmen erfüllen - Sanierungsmaßnahmen darstellten, dies jedoch verdeutlicht werden sollte.

- Drittens könnte in Erwägung gezogen werden, dass dem Begriff der Sanierungsmaßnahmen nach dem Erlass der RL 2014/59/EU keine eigene Bedeutung mehr zukommen solle bzw. der Anwendungsbereich der RL 2001/24/EG dadurch drastisch abgeändert worden sei.

In der Folge sollen diese drei Auslegungsmöglichkeiten analysiert werden, wobei zuerst auf die dritte Variante eingegangen wird, die sich recht rasch argumentativ entkräften lässt. Die Unterscheidung zwischen der ersten und der zweiten Variante spielt insofern eine bedeutende Rolle, als sie in Bezug auf die Einstufung der Maßnahmen rund um die Schaffung der HETA unterschiedliche Rechtsfolgen implizieren. Handelt es sich etwa nur um Abwicklungs-, aber keine Sanierungsmaßnahmen, so findet nur die RL 2014/59/EU und die RL 2001/24/EG in ihrer abgeänderten Form auf solche Maßnahmen Anwendung. Vertritt man hingegen die zweite Auslegungsvariante, so verstärkt dies die Ansicht, dass auch „typische“ Abwicklungsmaßnahmen bereits bislang in den Anwendungsbereich der RL 2001/24/EG fielen und von ihren Rechtswirkungen betroffen waren. ${ }^{114}$ Deutlich wird damit, dass die skizzierte Auslegungsfrage bzw. ihre Beantwortung für die Beurteilung, ob das GSA eine Sanierungsmaßnahme im Sinne der RL 2001/24/EG darstellt, ${ }^{115}$ von Bedeutung sind.

Obwohl im vorliegenden Beitrag bereits eine Einstufung des GSA als Abwicklungsmaßnahme erfolgte, bleiben die folgenden Ausführungen relevant. Einerseits gilt dies für den Fall, dass diesbezüglich eine andere Auffassung vertreten werden sollte. Andererseits kann so auch verdeutlicht werden, wie sich die Rechtslage gestaltet, wenn eine von den vorherigen Ausführungen zum persönlichen Anwendungsbereich der RL 2014/59/EU abweichende Meinung vertreten wird, d.h. die Abbaueinheit HETA als nicht unter den Begriff des Instituts der RL 2014/59/EU fallend eingestuft wird. Für diesen Fall klärt sich in den folgenden Ausführungen, ob womöglich aufgrund der Anwendbarkeit der RL 2001/24/EG das Vorgehen des österreichischen Gesetzgebers als Sanierungsmaßnahme(n) einzustufen ist und somit eine transnationale Rechtswirkung bzw. Anerkennungsverpflichtung in anderen Mitgliedstaaten sich direkt aus der Anwendung der RL 2001/24/EG in ihrer Fassung vor der Änderung durch RL 2014/59/EU ergibt.

aa) Zur These des Bedeutungsverlustes des Anwendungsbereichs von RL 2001/24/EG durch RL 2014/59/EU

In seiner neuen, durch RL 2014/59/EU abgeänderten ${ }^{116}$ Form sieht Art. 2 siebter Spiegelstrich RL 2001/24/EG vor, dass zu den darin definierten Sanierungsmaßnahmen ,auch“ die Anwendung der Abwicklungsinstrumente und die Ausübung der Abwicklungsbefugnisse gemäß der RL 2014/59/EU zählen.

Auf den ersten Blick könnte hier angenommen werden, dass typische Abwicklungsmaßnahmen wie die Maßnahmen, die bei der Schaffung der Abbaueinheit zum Einsatz kamen, nur unter diesen erweiterten Begriff der Sanierungsmaßnahmen fallen; falls

$114 \quad \mathrm{Zu}$ diesen bereits oben B.I.1.

115 Siehe unten B.III.2.b.

116 Art. 117 Abs. 2 RL 2014/59/EU. 
sie unter diesen Begriff nicht subsumiert werden können, wäre hingegen auch RL 2001/24/EG in ihrer neuen Fassung nicht anwendbar, d.h. insbesondere auch nicht die Verpflichtung zur Anerkennung derartiger Maßnahmen nach Art. 3 Abs. 2 RL 2001/24/EG. Nach diesem Ansatz käme jedoch seit Ablaufen der Umsetzungsfrist der RL 2014/59/EU Art. 2 siebter Spiegelstrich RL 2001/24/EG kein eigener Gehalt mehr zu. Dies überzeugt wenig, da bereits der Wortlaut der neu gefassten Bestimmung, nämlich der zweite Satz von Art. 2 siebter Spiegelstrich RL 2001/24/EG, darauf hinweist, dass zu „diesen Maßnahmen“, nämlich den Sanierungsmaßnahmen, ,auch“ Abwicklungsmaßnahmen gemäß der RL 2014/59/EU zählen. Somit erläutert die Neufassung der Bestimmung den Begriff der Sanierungsmaßnahmen näher und klärt, dass die Maßnahmen unter RL 2014/59/EU ebenfalls unter die RL 2001/24/EG fallen sollen und ihnen die Rechtswirkungen des Art. 3 Abs. 2 RL 2001/24/EG zukommen sollen. Hierfür - und gegen ein den Begriff der Sanierungsmaßnahmen der RL 2001/24/EG in neuer Fassung einschränkendes Verständnis - spricht darüber hinaus auch die Zielsetzung der RL 2014/59/EU, in der sich die relevante Änderung der RL 2001/24/EG findet. Die beiden Richtlinien sollen nämlich - wie bereits bei der Untersuchung des Verhältnisses der beiden Richtlinien erörtert ${ }^{117}$ - keineswegs einander einschränken oder widersprechen, sondern sich ergänzen und in ihrer Wirkung verstärken. Dem entspräche eine den Anwendungsbereich der RL 2001/24/EG in neuer Fassung einschränkende Auslegung des Begriffs der Sanierungsmaßnahmen nach Ablauf der Umsetzungsfrist der RL 2014/59/EU nicht.

bb) Zur These der Ergänzung des Anwendungsbereiches von RL 2001/24/EG durch RL 2014/59/EU

Als weitere Lesart kommt in Frage, die Änderung des Art. 2 siebter Spiegelstrich RL 2001/24/EG als Ergänzung zu lesen, d.h. dass eine neue Kategorie von Maßnahmen dem bisherigen Kreis von Sanierungsmaßnahmen hinzugefügt werde.

Als Sanierungsmaßnahmen im Sinne der RL 2001/24/EG definierte Art. 2 siebter Spiegelstrich ursprünglich Maßnahmen, ,mit denen die finanzielle Lage eines Kreditinstituts gesichert oder wiederhergestellt werden soll und die die bestehenden Rechte Dritter beeinträchtigen könnten“ und nennt als Beispiele demonstrativ ${ }^{118}$ Aussetzungen von Zahlungen, von Vollstreckungsmaßnahmen oder Kürzungen von Forderungen. Auch die neue, durch die RL 2014/59/EU geänderte Fassung behält diese Definition weitgehend bei und fügt lediglich hinzu, dass $\mathrm{zu}$ diesen Maßnahmen auch die Anwendung der Abwicklungsinstrumente und die Ausübung der Abwicklungsbefugnisse der RL 2014/59/EU gehören. ${ }^{119}$

Fraglich ist, ob dem Begriff der Sanierungsmaßnahmen gemäß der RL 2001/24/EG in alter Fassung eine weite oder enge Auslegung zugrunde gelegt werden soll. Gemäß einer engen Auffassung wären nur Maßnahmen erfasst, die zur Stärkung bzw. Gesundung eines Institutes beitragen. Eine weite, keine streng formale Betrachtung zugrunde legende Auffassung bezieht im Lichte des Wortlautes von Art. 2 siebter Spiegelstrich RL 2001/24/EG auch Maßnahmen mit ein, die eher einem dynamischen Ansatz folgend der Stabilisierung eines Institutes dienen. ${ }^{120} \mathrm{Zu}$ weit dürfte es hingegen jedenfalls gehen, wenn nicht mehr das Bestehen eines Institutes sichergestellt werden soll, sondern Maßnahmen nur noch mit dem Ziel getroffen

\footnotetext{
117 B.I.1.

118 Die Bestimmung verwendet den Begriff „einschließlich“.

119 Art. 117 Abs. 2 RL 2014/59/EU.

120 Lehmann/Hoffmann, Bankenrestrukturierung mit Hindernissen: Die Übertragung im Ausland belegener systemrelevanter Funktionen, WM 2013, S. 1389 ff. (S. 1389).
} 
werden, unerwünschte Auswirkungen bei der Abwicklung, d.h. Zerschlagung oder Auflösung, eines Institutes zu verhindern. Nur unter Zugrundelegung einer solchen Auslegung würde jedoch der Anwendungsbereich der RL 2001/24/EG durch RL 2014/59/EU nicht erweitert, die ,,auch“ Abwicklungsmaßnahmen zu Sanierungsmaßnahmen erklärt.

Letztlich soll gemäß dem Wortlaut der Bestimmung der RL 2001/24/EG nach alter Fassung die finanzielle Lage eines Kreditinstitutes ,gesichert oder wiederhergestellt“ werden, was reine Abbaumaßnahmen nicht mehr erfassen dürfte. In diesem Lichte kann auch nicht formal auf die Natur einer Maßnahme abgestellt werden, sondern sie ist stets im Lichte des verfolgten Ziels zu untersuchen. Auch eine Übertragung von Unternehmensteilen kann so je nach Zielsetzung dem Abbau des Institutes oder der Gesundung dienen.

Nach dieser vom Wortlaut klarer gedeckten und somit letztlich überzeugenderen Lesart sind Sanierungsmaßnahmen im Sinne der RL 2001/24/EG nach alter Fassung von der Zielsetzung getragen, die finanzielle Lage eines Institutes direkt oder indirekt zu stabilisieren oder zu verbessern, nicht jedoch von jener, ein Institut mit möglichst geringen Folgewirkungen abzubauen. Die RL 2014/59/EU ergänzt aus diesem Grund den Anwendungsbereich der RL 2001/24/EG, wenn sie in Art. 2 siebter Spiegelstrich RL 2001/24/EG Abwicklungsmaßnahmen als eigene Kategorie den Sanierungsmaßnahmen hinzufügt und damit die Gruppe der Sanierungsmaßnahmen erweitert.

Dementsprechend ist also zwischen Sanierungsmaßnahmen im Sinne der RL 2001/24/EG alter Fassung und Abwicklungsmaßnahmen im Sinne der RL 2014/59/EU zu unterscheiden. Letztere fallen zwar auch aufgrund des zweiten Satzes, der durch diese Richtlinie zu Abs. 2 siebter Spiegelstrich RL 2001/24/EG hinzugefügt wird, in den Anwendungsbereich der RL 2001/24/EG, was u.a. die Rechtsfolgen des Art. 3 Abs. 2 RL 2001/24/EG auslöst. In den weiter zu verstehenden Kreis der Sanierungsmaßnahmen nach RL 2001/24/EG fallen jedoch auch Maßnahmen, die keine Abwicklungsmaßnahmen sind und für die daher nur die Vorgaben RL 2001/24/EG gelten. ${ }^{121}$

cc) Zur These der Konkretisierung des Anwendungsbereiches von RL 2001/24/EG durch RL 2014/59/EU

Als Gegenthese ließe sich vertreten, die Änderungen durch die RL 2014/59/EU an RL 2001/24/EG seien rein klarstellender Natur. Insbesondere wenn eine sehr weit gefasste Auslegung des von der RL 2001/24/EG in alter Fassung geprägten Begriffs der Sanierungsmaßnahmen vertreten wird, würden Abwicklungsmaßnahmen, die die Mitgliedstaaten vor Ablauf der Umsetzungsfrist der RL 2014/59/EU ergriffen haben, auch als Sanierungsmaßnahmen bereits weitgehend in den Anwendungsbereich der RL 2001/24/EG alter Fassung fallen. ${ }^{122}$ Zumindest aufgrund des Wortlautes des Art. 2 siebter Spiegelstrich RL 2001/24/EG in neuer Fassung, demzufolge $\mathrm{zu}$ den darin definierten Sanierungsmaßnahmen ,auch“ die Anwendung der Abwicklungsinstrumente und die Ausübung der Abwicklungsbefugnisse gemäß der RL 2014/59/EU zählen, könnte vertreten werden, dass nur eine beispielhafte Konkretisierung des Begriffs der Sanierungsmaßnahmen erfolgte.

Hingegen überzeugt dies aus mehreren Gründen wenig. So enthält Art. 2 siebter Spiegelstrich RL 2001/24/EG bereits in alter Fassung eine Aufzählung von Maßnahmen als Beispiele für Sanierungsmaßnahmen. In diese Beispielliste hätten für den Fall, dass diese den Begriff der

121 So auch EuGH, Rs. C-526/14 (Kotnik), EU:C:2016:570, Rdnr. 113, bzw. ausführlich Schlussanträge des Generalanwalts Wahl, EuGH, Rs. C-526/14 (Kotnik), EU:C:2016:102, Rdnr. 143. Hierzu noch näher B.III.2.b. am Beispiel der Schaffung der Abbaueinheit HETA. 
Sanierungsmaßnahmen nur konkretisieren sollte und Sanierungsmaßnahmen ohnedies typische Abwicklungsmaßnahmen umfassen, Sanierungsmaßnahmen aufgenommen werden können. Darüber hinaus entschied sich der Gesetzgeber, die Abwicklungsmaßnahmen in einen eigenen Satz mit dem Wort ,auch“ als zu den Sanierungsmaßnahmen zählend einzuführen. Dies weist darauf hin, dass sie zuvor nicht vom Begriff der Sanierungsmaßnahmen erfasst waren, da ein eigener ausdrücklicher Hinweis als erforderlich erachtet wurde, dass sie ebenso ab Inkrafttreten der RL 2014/59/EU als neue Kategorie unter den bisher anders bzw. enger definierten Begriff der Sanierungsmaßnahmen im Sinne der RL 2001/24/EG alter Fassung fallen.

Somit vermag die These einer rein konkretisierenden Wirkung der Änderungen der RL 2014/59/EU in Bezug auf den Anwendungsbereich der RL 2001/24/EG letztlich nicht zu überzeugen. Vielmehr bestand vor Erlass der RL 2014/59/EU bereits ein eigenständiger Begriff der Sanierungsmaßnahmen in der RL 2001/24/EG. Dessen Definition, die als solche weiterhin aufgrund des diesbezüglich gleich bleibenden Wortlautes von Art. 2 siebter Spiegelstrich bestehen bleibt, wird lediglich um Sanierungsmaßnahmen erweitert, die aufgrund der entsprechenden Anordnung in Art. 2 siebter Spiegelstrich zweiter Satz RL 2001/24/EG n.F. als Sanierungsmaßnahmen zu verstehen sind.

Somit ist im Ergebnis das Verhältnis zwischen den beiden Anwendungsbereichen so zu verstehen, dass in Bezug auf Abwicklungsmaßnahmen die RL 2014/59/EU soweit zur Anwendung kommt, als sie für derartige Maßnahmen das speziellere Regelwerk darstellt. Soweit eine Maßnahme nicht unter die RL 2014/59/EU fällt, kann das allgemeinere Regelwerk der RL 2001/24/EG anwendbar sein, sofern es sich um eine Sanierungsmaßnahme handelt. Jedoch besteht in Bezug auf die Rechtswirkungen des Art. 3 Abs. 2 RL 2001/24/EG, wie sie nach Abänderung durch die RL 2014/59/EU zum Zuge kommen, kein Widerspruch zu den Vorgaben der RL 2014/59/EU. Abwicklungsmaßnahmen darf und soll die Wirkung des Art. 3 Abs. 2 RL 2001/24/EG zukommen, nämlich eine Verpflichtung zur Anerkennung derartiger Maßnahmen in anderen Mitgliedstaaten.

Eine genauere Analyse der RL 2001/24/EG und 2014/59/EU bzw. der Änderungen, die letztere an ersterer vorgenommen hat, zeigt, dass weiterhin zur Feststellung des Anwendungsbereichs der RL 2001/24/EG zuerst auf den bereits in der alten Fassung vorhandenen Begriff der Sanierungsmaßnahmen abzustellen ist. Dabei handelt es sich um auf die Sicherung oder Wiederherstellung der finanziellen Lage eines Instituts abzielende Maßnahmen. Die RL 2014/59/EU fügt dem Begriff der Sanierungsmaßnahmen die Kategorie der Abwicklungsmaßnahmen aus der RL 2014/59/EU hinzu, die nunmehr als Teil eines erweiterten Begriffes der Sanierungsmaßnahmen zu verstehen sind, denen allen die in RL 2001/24/EG vorgesehenen Rechtswirkungen zukommen, so insbesondere die Anerkennungspflicht in anderen Mitgliedstaaten nach Art. 3 Abs. 2 RL 2001/24/EG.

\section{b) Zum GSA und zum Begriff der Sanierungsmaßnahme im Sinne der RL 2001/24/EG nach alter Fassung}

Wie erläutert, definiert Art. 2 siebter Spiegelstrich RL 2001/24/EG Sanierungsmaßnahmen als jene Maßnahmen, „mit denen die finanzielle Lage eines Kreditinstituts gesichert oder wiederhergestellt werden soll und die die bestehenden Rechte Dritter beeinträchtigen 
könnten“ und nennt als Beispiele demonstrativ ${ }^{123}$ Aussetzungen von Zahlungen, von Vollstreckungsmaßnahmen oder Kürzungen von Forderungen. Auch die neue, durch die RL 2014/59/EU geänderte Fassung behält diese Definition grundsätzlich bei und fügt nur hinzu, dass zu diesen Maßnahmen auch die Anwendung der Abwicklungsinstrumente und die Ausübung der Abwicklungsbefugnisse der RL 2014/59/EU gehören. ${ }^{124}$ Wie erörtert, ${ }^{125}$ werden dadurch Abwicklungsmaßnahmen zu Sanierungsmaßnahmen erklärt und der Kreis letzterer erweitert, ohne dass jedoch dabei der ursprüngliche Anwendungsbereich der RL 2001/24/EG beschränkt wird. Obwohl nunmehr bereits das GSA grundsätzlich als Abwicklungsmaßnahme im Sinne der RL 2014/59/EU eingestuft wurde, ist an dieser Stelle zusätzlich zu untersuchen, ob das GSA oder Teile davon auch als Sanierungsmaßnahme eingestuft werden können. Ist dies der Fall, kommt solchen Teilen die Rechtswirkung insbesondere von Art. 3 Abs. 2 RL 2001/24/EG zu. Insbesondere käme solchen Teilen auch dann die entsprechende Rechtswirkung zu, wenn - entgegen der hier vertretenen Ansicht ${ }^{126}$ - ein engerer Ansatz zum Anwendungsbereich der RL 2014/59/EU vertreten würde, der die Schaffung der HETA als Abbaueinheit kategorisch von besagtem Anwendungsbereich ausschlösse.

Einleitend sind an dieser Stelle einige allgemeine Feststellungen zum Begriff der Sanierungsmaßnahmen in RL 2001/24/EG zu treffen (aa), bevor das GSA in diesem Lichte eingeordnet wird (bb).

\section{aa) Zum Begriff der „Sanierungsmaßnahme“ in der RL 2001/24/EG}

Als zentrales Element wurde bereits festgestellt, ${ }^{127}$ dass aufgrund des Wortlautes Sanierungsmaßnahmen im Sinne der RL 2001/24/EG a.F. primär von der Zielsetzung getragen sein müssen, die finanzielle Lage eines Institutes direkt oder indirekt zu stabilisieren oder $\mathrm{zu}$ verbessern, nicht jedoch von jener, ein Institut mit möglichst geringen Folgewirkungen abzubauen, da es sich in einem solchen Fall um Abwicklungsmaßnahmen handeln würde. ${ }^{128}$

Bereits in Bezug auf den persönlichen Anwendungsbereich der RL 2001/24/EG ist hervorzuheben, dass diese die Frage des Verlusts der Zulassung im Vergleich zur RL 2014/59/EU klarer regelt. Deutlicher noch als bei RL 2014/59/EU kommt beim persönlichen Anwendungsbereich der RL 2001/24/EG nämlich zum Ausdruck, dass ein Kreditinstitut auch beim Verlust einer Bankenzulassung weiterhin in den Anwendungsbereich der Richtlinie fällt. Art. 12 RL 2001/24/EG sieht dies ausdrücklich für den Fall eines Liquidationsverfahrens vor. Aber auch beim Sanierungsverfahren, das in Art. 3 ff. RL 2001/24/EG geregelt ist, weist nichts im Wortlaut oder aber auch in den Zielsetzungen der Richtlinie darauf hin, dass die Anwendbarkeit der Vorgaben vom Bestehen einer Bankenkonzession abhängig gemacht wird. ${ }^{129}$ Ein solches Vorgehen entspräche wohl auch nicht den Zielsetzungen der RL 2001/24/EG, die vorsehen, dass bei Sanierungsmaßnahmen und Liquidationsverfahren primär sicherzustellen ist, dass ein Kreditinstitut und seine Zweigstellen zu diesen Zwecken als Einheit behandelt werden sollten. ${ }^{130}$

Unter den Begriff der Sanierungsmaßnahmen fallen dem Wortlaut zufolge jene Maßnahmen, mit denen die finanzielle Lage eines Institutes ,gesichert oder wiederhergestellt werden soll“.

123 Die Bestimmung verwendet den Begriff, „einschließlich“.

124 Art. 117 Abs. 2 RL 2014/59/EU.

125 Oben B.III.2.a.

126 Oben B.II.

127 Oben B.III.2.a.

128 Siehe auch EuGH, Rs. C-526/14 (Kotnik), EU:C:2016:570, Rdnr. 108 f.

129 Etwa Erw. 13, 14 oder 19 RL 2001/24/EG.

130 Erw. 4 RL 2001/24/EG. 
Auch in Anbetracht dieses eher weit gefassten Wortlautes drängt sich ein dynamisches, breites Verständnis dieses Begriffes auf, der somit wohl auch Maßnahmen umfasst, die der Gesetzgeber beim Erlass der RL 2001/24/EG noch selbst nicht unbedingt im Detail vorhersehen konnte. Für diesen Ansatz spricht auch, dass sowohl Maßnahmen der Wiederherstellung der finanziellen Lage als auch solche der reinen Sicherung erwähnt werden. Damit sind wohl auch Maßnahmen erfasst, die lediglich den aktuellen Zustand bewahren und eine Verschlimmerung der Lage verhindern sollen.

Demgegenüber sind a priori denkbare beschränkende Lesarten des Begriffs der Sanierungsmaßnahmen wenig überzeugend:

- So könnte zwar a priori argumentiert werden, nur bestimmte „traditionelle“ Sanierungsmaßnahmen seien als ebensolche anzuerkennen. Diesbezüglich fehlt es jedoch bereits an einer solchen Definition der Sanierungsmaßnahmen im Wortlaut der Richtlinie. Darüber hinaus ist die Zielsetzung der Richtlinie, das Herkunftslandsprinzip für Sanierungsmaßnahmen einzuführen bzw. die Anerkennung solcher Sanierungsmaßnahmen in anderen Mitgliedstaaten sicherzustellen und nicht eine vereinheitlichende Definition solcher Maßnahmen zu schaffen. ${ }^{131}$ Eben daraus ergibt sich, dass die Mitgliedstaaten über einen gewissen Gestaltungsspielraum bei der Festlegung von Sanierungsmaßnahmen verfügen und mit Sanierungsmaßnahmen keine eingeschränkte Gruppe von „typischen“ oder „traditionellen“ Sanierungsmaßnahmen gemeint sein können.

- $\quad$ Als weitere denkbare Einschränkung könnte man vertreten, nur Maßnahmen eines gewissen Umfangs bzw. mit einer gewissen Mindestwirkung könnten als Sanierungsmaßnahmen angesehen werden. Auch eine solche Auffassung findet jedoch keine Stütze im Wortlaut, enthält dieser doch eine offene Aufzählung von Beispielen ohne nähere Qualifikation. ${ }^{132}$ Zugleich liefe es auch der Zielsetzung der Richtlinie entgegen, nur Maßnahmen einer gewissen Schwere als Sanierungsmaßnahmen anzuerkennen, könnten doch so Mitgliedstaaten entgegen der Grundidee der Richtlinie erneut die Anerkennung von Sanierungsmaßnahmen in anderen Mitgliedstaaten mit eben dem Argument ablehnen, eine Maßnahme sei nicht gewichtig genug.

- Darüber hinaus zeigt sich an der Systematik der Richtlinie, dass mit Sanierungsmaßnahmen eine breite Palette von Maßnahmen gemeint ist. In Art. 12 RL 2001/24/EG wird nämlich als einzige Form von Schwelle festgehalten, dass als Teil der Einleitung eines Liquidationsverfahrens die Zulassung eines Institutes zu entziehen ist. Bis zu jenem Zeitpunkt hingegen gewährt die Richtlinie einen weiten Spielraum, heisst es doch, dass ein solcher Entzug auch $\mathrm{zu}$ beschliessen sei, ohne dass Sanierungsmaßnahmen getroffen wurden oder nachdem diese gescheitert sind. ${ }^{133}$ Auch das allgemeine System der Richtlinie zielt somit nicht auf eine irgendwie geartete Definition von Sanierungsmaßnahmen ab, sondern legt als einzig klaren Orientierungspunkt den Entzug der Zulassung bei Einleitung eines Liquidationsverfahrens fest.

- Denkbar wäre schließlich, die Definition von Sanierungsmaßnahmen einzuschränken, da in Art. 3 Abs. 1 RL 2001/24/EG nur „die Behörden und Gerichte“ des Herkunftsmitgliedstaates befugt sind, über die Durchführung solcher Maßnahmen zu entscheiden. Dementsprechend urteilte etwa das LG München in einer einschlägigen Entscheidung, gesetzliche Maßnahmen könnten keine Sanierungsmaßnahmen

$131 \quad$ Erw. 7 RL 2001/24/EG.

132 Etwa hätte der Unionsgesetzgeber eine „umfassende“ oder „,bedeutende“ Kürzung von Forderungen als Beispiel nennen können, hat sich jedoch offenbar bewusst dagegen entschieden. 
darstellen. ${ }^{134}$ Als Folge könnte auch in Frage gestellt werden, ob das GSA als gesetzliche Maßnahme überhaupt in den Anwendungsbereich der RL 2001/24/EG fallen kann. Jedoch ist eine solche Einschränkung des Begriffs der Sanierungsmaßnahmen gemäß RL 2001/24/EG bei näherer Betrachtung wenig überzeugend. Zwar verwendet Art. 3 Abs. 1 RL 2001/24/EG diese Formulierung; jedoch stützt die für „Behörden oder Gerichte“ in der Richtlinie enthaltene Definition kaum eine einschränkende Lesart. Als solche Institutionen gelten nämlich ,die Behörden oder Gerichte der Mitgliedstaaten, die für Sanierungsmaßnahmen oder Liquidationsverfahren zuständig sind““. ${ }^{135}$ Aus der Zirkeldefinition ergibt sich bereits, dass das primäre Merkmal derartiger Behörden oder Gerichte nicht so sehr deren Zugehörigkeit zu einer bestimmten Staatsgewalt, sondern deren Zuständigkeit sein soll. Dieses Verständnis wird durch die Zielsetzung der Richtlinie bestätigt. Es soll nämlich vor allem die Wirksamkeit von Sanierungsmaßnahmen in allen Mitgliedstaaten sichergestellt werden, ob nun Maßnahmen von Behörden oder Gerichten eines Mitgliedstaates ergriffen werden oder diese „Personen oder Organe“ mit der Durchführung solcher Maßnahmen beauftragen. ${ }^{136}$ Dafür sind breit gefasst die Maßnahmen anzuerkennen, „die ein einzelner Mitgliedstaat trifft" - ohne Differenzierung, um welche handelnde Staatsgewalt eines Mitgliedstaats es sich im Detail handelt. ${ }^{137}$ Auch soll so vorgegangen werden, eben weil „die Harmonisierung der Rechtsvorschriften und Gepflogenheiten der Mitgliedstaaten schwierig“ sei; ${ }^{138}$ gerade vor diesem Hintergrund erscheint es problematisch, wenn nunmehr die Begriffe der „Behörden oder Gerichte“ eng ausgelegt werden sollen. Die Richtlinie zielt eben darauf ab, die Vielfalt der Regulierungsansätze der Mitgliedstaaten in Bezug auf Sanierungsmaßnahmen als gegeben hinzunehmen und konzentriert sich auf die Wirkung der jeweiligen Maßnahmen in anderen Mitgliedstaaten. Dem liefe es nun zuwider, ohne Grundlage strenge Voraussetzungen in der Richtlinie anzunehmen, in welchem Ausmaß die einzelnen Staatsgewalten eines Mitgliedstaates an einer Sanierungsmaßnahme beteiligt sein müssen. Somit können im Ergebnis wohl auch Maßnahmen, die ein Tätigwerden von Gesetzgeber und Verwaltungsbehörden umfassen, als Sanierungsmaßnahmen anerkannt werden.

bb) Zur Einstufung des GSA als „Sanierungsmaßnahme“

Auf der Grundlage des dargelegten weiten Verständnisses des Begriffs der Sanierungsmaßnahme in der RL 2001/24/EG sind nun die einzelnen Elemente des GSA zu prüfen:

- Die Ermächtigung des Bundesministers der Finanzen, eine Aktiengesellschaft, die „ABBAG - Abbaubeteiligungsaktiengesellschaft des Bundes“, zu errichten und die Anteile an der Hypo Alpe-Adria-Bank International AG bzw. der Abbaueinheit und die damit zusammenhängenden Forderungen und Verbindlichkeiten in besagte Aktiengesellschaft einzubringen, ${ }^{139}$ könnte als Sanierungsmaßnahme eingestuft

Siehe hierzu und näher zur Entscheidung Rühle/Schmitz, Keine Anerkennung ausländischer Eingriffsnormen ohne Rechtsgrundlage für die Wirkungserstreckung - LG München I, Urteil vom 8.5.2015 - 32 O 26502/12, BeckRS 2015, 15096, S. 406 f. (S. 407). Art. 2 sechster Spiegelstrich RL 2001/24/EG.

Erw. 7 RL 2001/24/EG.

Erw. 6 RL 2001/24/EG.

Erw. 6 RL 2001/24/EG.

$\S 2$ Abs. 5 GSA. 
werden. Hier wäre zu prüfen, ob diese rechtsgeschäftliche Übertragung von Anteilen in eine eigene Aktiengesellschaft primär dem Ziel der Stabilisierung dient oder aber eng mit der später erfolgenden Abwicklung in Form der errichteten Abbaueinheit verbunden ist, eine Analyse, die hier jedoch nicht geleistet werden kann.

- $\quad$ Die Vorgabe des GSA, die Abbaueinheit möge die eigenen Vermögenswerte im Rahmen des Portfolioabbaus so verwalten, dass eine ,geordnete, aktive und bestmögliche Verwertung“ sichergestellt sei, ${ }^{140}$ dient hingegen nur mehr der optimalen Abwicklung und nicht mehr in irgendeiner Form der Stabilisierung oder Verbesserung der Lage des betroffenen Unternehmens.

- $\quad$ Ähnlich fällt die Beurteilung der Bestimmung aus, mit der das GSA den Bundesminister für Finanzen ermächtigt, durch Verordnung zur Schaffung einer Abbaueinheit Teile der Hypo Alpe-Adria-Bank International AG auf den Bund oder einen anderen aufnehmenden Rechtsträger gegen angemessenes Entgelt auszugliedern, ${ }^{141}$ auch wenn der Erwerb von Vermögenswerten ggf. auch rechtsgeschäftlich erfolgen kann. ${ }^{142}$ Das GSA bestimmt zusätzlich, dass, soweit Gläubigern der Hypo Alpe-Adria-Bank International AG Kündigungs-, Zustimmungs- oder andere Gestaltungsrechte oder Rechte auf Sicherstellung ihrer Forderungen zukommen, diese im Fall einer solchen Übertragungsanordnung oder auch einer rechtsgeschäftlichen Übertragung ,nicht anzuwenden und nicht ausübbar" seien. ${ }^{143}$ Zwar lässt die zweite Bestimmung durchaus an eine Sanierungsmaßnahme denken, da durch die Einschränkung der Geltendmachungsmöglichkeiten der Ansprüche der Gläubiger ein Beitrag zur Stabilisierung eines Unternehmens geleistet wird. Jedoch ist diese Maßnahme direkt verbunden mit der Schaffung einer Abbaueinheit und der Ausgliederung von Vermögenswerten des betroffenen Unternehmens. Bei einer solchen Ausgliederung könnte zwar theoretisch auch die Stabilisierung eines Unternehmens im Mittelpunkt stehen. Praktisch ist jedoch der Zweck des GSA bei der Ausgliederung eindeutig, eine Abbaueinheit zu schaffen und nicht die Sicherung der finanziellen Situation der Hypo

Alpe-Adria-Bank International AG. Somit können beide Maßnahmen in ihrer Gesamtheit wohl nur als Abwicklungsmaßnahmen verstanden werden, und eine Einstufung als Sanierungsmaßnahme scheidet aus.

Trotz der zuvor erörterten weiten Definition von Sanierungsmaßnahmen in RL 2001/24/EG zeigt sich somit, dass die einzelnen im GSA getroffenen Maßnahmen mit der Abwicklung der Hypo Alpe-Adria-Bank International AG befasst sind und nicht mit der Sicherung oder gar Verbesserung ihrer finanziellen Situation. Einzig die Übertragung von Anteilen der Bank in eine eigene Aktiengesellschaft könnte bei entsprechender Beurteilung aus ökonomischen Gesichtspunkten diese Voraussetzung erfüllen.

\section{Zu den Rechtswirkungen des Moratoriums der FMA}

Auf der Grundlage der bisherigen Ausführungen stellt sich in Bezug auf das Moratorium der FMA als möglicherweise unionsrechtlich geregelte Abwicklungsmaßnahme im Sinne der RL 2001/24/EG und 2014/59/EU die Frage der Rechtsfolgen dieser Maßnahme, insbesondere in

\begin{tabular}{ll}
\hline 140 & $\S 3$ Abs. 1 GSA. \\
141 & $\$ 1$ Abs. 1 GSA. \\
142 & $\S 1$ Abs. 5 GSA. \\
143 & $\S 1$ Abs. 4 GSA.
\end{tabular}


Bezug auf eine mögliche unionsrechtliche Verpflichtung zur Anerkennung der Rechtswirkung eines solchen Moratoriums für die Gerichte anderer Mitgliedstaaten.

Am 1. März 2015 erließ die FMA einen Bescheid (im Folgenden als Moratorium bezeichnet), in dem angeordnet wurde, dass der Fälligkeitszeitpunkt der vom Bescheid erfassten Verbindlichkeiten mit sofortiger Wirkung sowohl für die betroffenen Gläubiger als auch für die HETA auf den 31. Mai 2016 hinausgeschoben wird. Im Entscheid begründet die FMA die Verhältnismäßigkeit der getroffenen Maßnahme und stützt diese auf $\S 50$ Abs. 1 Z 2 i.V.m. $§ 58$ Abs. 1 BaSAG. Ziel sei es, eine ungerechtfertigte bevorzugte Befriedigung einzelner Gläubiger bzw. ein finanzielles „Ausbluten“ der HETA und andere Schäden zu verhindern, die etwa durch Verhandlungen unter Zeitdruck verursacht werden könnten. Der Bescheid enthält keine näheren Angaben zu seiner sachlichen, persönlichen oder räumlichen Wirkung, und insbesondere nicht zur Wirkung in Bezug auf in anderen Mitgliedstaaten durch die HETA eingegangene Rechtsverhältnisse. ${ }^{144}$

Nachfolgend kann auf die bereits angestellten Abklärungen zum persönlichen Anwendungsbereich der RL 2014/59/EU ${ }^{145}$ und zum Verhältnis der sachlichen Anwendungsbereiche der RL 2014/59/EU und 2001/24/EG ${ }^{146}$ zurückgegriffen werden. Bejaht man auf dieser Grundlage, dass die Schaffung der Abbaueinheit und somit die HETA in den Anwendungsbereich der RL 2014/59/EU fällt, ${ }^{147}$ so ist die Einstufung des Moratoriums als Abwicklungsmaßnahme im Sinne der RL 2014/59/EU zu untersuchen (I.). Im Fall einer solchen Einstufung kommt dem Moratoriumsbescheid die Rechtswirkung von Art. 3 Abs. 2 RL 2001/24/EG zu, d.h. es besteht eine unionsrechtliche Verpflichtung, derartige Abwicklungsmaßnahmen im nationalen Recht sämtlicher Mitgliedstaaten anzuerkennen und ihnen vollumfänglich Wirkung zukommen zu lassen. ${ }^{148}$ Selbst wenn man die Anwendung der RL 2014/59/EU verneint, bleibt aber zu prüfen, ob das Moratorium als Sanierungs maßnahme im Sinne der RL 2001/24/EG einzustufen ist, da auch in einem solchen Fall aufgrund von Art. 3 Abs. 2 RL 2001/24/EG dieselben Rechtswirkungen einträten (II.).

\section{Zur Einstufung des Moratoriums als Abwicklungsmaßnahme im Sinne der RL 2014/59/EU}

Damit das Moratorium der FMA als Abwicklungsmaßnahme eingestuft werden kann, muss es von einer Abwicklungsbehörde im Sinne der RL 2014/59/EU angeordnet worden sein und sich materiell als Abwicklungmaßnahme einstufen lassen.

Die FMA ist zweifelsohne, wie in der Richtlinie vorgesehen, in korrekter Weise vom österreichischen Gesetzgeber als Abwicklungsbehörde vorgesehen worden. Art. 3 Abs. 3 RL 2014/59/EU sieht vor, dass die Mitgliedstaaten als solche Abwicklungsbehörden nationale Zentralbanken, die zuständigen Ministerien oder andere öffentliche Verwaltungsbehörden oder Behörden, denen Befugnisse der öffentlichen Verwaltung übertragen wurden, benennen können.

Als Teil der Abwicklungsbefugnisse einer Abwicklungsbehörde sieht Art. 63 Abs. 1 Bst. j RL 2014/59/EU vor, dass letztere die Befugnis haben müssen, „die Fälligkeit der von einem

144 Darauf hinzuweisen ist, dass in der Zwischenzeit die Finanzmarktaufsicht am 10.4.2016 den Mandatsbescheid vom 1. März 2015 in einem Vorstellungsbescheid bestätigt und einen weiteren Mandatsbescheid erlassen hat, in dem sie Abwicklungsmaßnahmen nach dem BaSAG angewendet hat. Die entsprechenden Bescheide können unter www.heta-asset-resolution.com eingesehen werden (zuletzt eingesehen am 14.11.2016). Oben B.II.

Oben B.III.

Vgl. die Gegenposition bei Fest (Fußn. 7), S. 1111.

Siehe hierzu bereits B.II.1. 
in Abwicklung befindlichen Institut ausgegebenen Schuldtitel und anderen berücksichtigungsfähigen Verbindlichkeiten oder den aufgrund der entsprechenden Schuldtitel und anderen berücksichtigungsfähigen Verbindlichkeiten zahlbaren Zinsbetrag oder den Zeitpunkt, an dem die Zinsen zu zahlen sind, zu ändern, und zwar auch durch eine zeitlich befristete Aussetzung der Zahlungen“. Eben eine solche Befugnis wird im Rahmen des Moratoriums durch die FMA in Form ihres Bescheides ausgeübt: Das Fälligkeitsdatum der vom Bescheid erfassten Verbindlichkeiten wird für die Gläubiger und für die HETA um eine begrenzte Zeitspanne ausgedehnt.

Als einzige Ausnahme sieht Art. 63 Abs. 1 Bst. j RL 2014/59/EU vor, dass sich diese Befugnis nicht auf „,besichert[e] Verbindlichkeiten im Sinne des Artikels 44 Absatz 2“ erstreckt. Zu prüfen ist an dieser Stelle, ob unter diesen Begriff jene vom Moratorium erfassten Verbindlichkeiten fallen, für die eine gesetzliche Haftung besteht. Bereits die Auflistung des Art. 44 Abs. 2 RL 2014/59/EU weist jedoch nicht in die Richtung von solchen, durch eine gesetzliche Haftung gedeckten Verbindlichkeiten, spricht die Bestimmung doch in ihrer Auflistung beispielsweise von Verbindlichkeiten gegenüber Beschäftigten aufgrund ausstehender Lohnforderungen ${ }^{149}$ oder allgemeiner von ,gedeckten Schuldverschreibungen und Verbindlichkeiten in Form von Finanzinstrumenten, die zu Absicherungszwecken verwendet werden, die einen festen Bestandteil des Deckungsstocks bilden und die nach einzelstaatlichem Recht ähnlich wie gedeckte Schuldverschreibungen besichert sind“. ${ }^{150}$ Dies deutet darauf hin, dass hier typischerweise das besondere Vertrauen geschützt werden soll, das entsteht, wenn ein Gläubiger mit einem Schuldner eine Sicherung vereinbart. Für diesen Ansatz spricht auch die Definition der „besicherten Verbindlichkeit“ in den Begriffsbestimmungen der RL 2014/59/EU. Dieser Definition zufolge handelt es sich nämlich dabei um eine Verbindlichkeit, ,,bei der der Anspruch des Gläubigers auf Zahlung [...] durch ein Pfand oder pfandrechtsähnliches Zurückbehaltungsrecht oder durch eine Sicherungsvereinbarung abgesichert ist". Erneut wird klar, dass unter diesen auf Vereinbarungen bezogenen Begriff der besicherten Verbindlichkeiten nicht die einseitig gesetzlich angeordnete Haftung eines öffentlichen Rechtsträgers fällt. Somit handelte die FMA auch in Bezug auf die vom Moratorium erfassten Verbindlichkeiten im Sinne der RL 2014/59/EU.

Zuletzt könnte in Erwägung gezogen werden, das Moratorium der FMA deshalb nicht als Abwicklungsmaßnahme im Sinne der RL 2014/59/EU anzusehen, weil es an einem ausreichend unmittelbaren Zusammenhang zwischen dem Einsatz von Abwicklungsinstrumenten und der in Form des Moratoriums vorliegenden Ausübung einer Abwicklungsbefugnis im Sinne der RL 2014/59/EU mangelt. Allerdings findet sich keine Vorgabe in der Richtlinie, die einen solchen unmittelbaren Zusammenhang oder Bezug fordert. Art. 63 Abs. 1 RL 2014/59/EU verpflichtet die Mitgliedstaaten in Bezug auf Abwicklungsbefugnisse nur sicherzustellen, dass die Abwicklungsbehörden über sämtliche Befugnisse verfügen, ,um die Abwicklungsinstrumente auf Institute [...] anzuwenden, die die geltenden Voraussetzungen für eine Abwicklung erfüllen“. Der genaue Zusammenhang zwischen Abwicklungsbefugnissen und -instrumenten wird damit aber nicht angesprochen, ganz zu schweigen von der Formulierung eines irgendwie gearteten Erfordernisses eines unmittelbaren Zusammenhangs zwischen dem Einsatz von Abwicklungsinstrumenten und der in Form des Moratoriums vorliegenden Ausübung einer Abwicklungsbefugnis. Im Gegenteil zeigt sich bereits anhand der verschiedenen, in der Folge aufgeführten Abwicklungsbefugnisse, dass diese teils zur unmittelbaren Anwendung eines 
Abwicklungsinstrumentes, ${ }^{151}$ in anderen Fällen jedoch eher zur Vorbereitung des späteren Einsatzes eines Abwicklungsinstrumentes dienen sollen. ${ }^{152}$ Auch im Rahmen einer teleologischen Betrachtung überzeugt es nicht, hier einen unmittelbaren Zusammenhang zwischen Befugnis und Instrument zu verlangen. Anderenfalls müsste nämlich eine Behörde bereits bei der Anwendung selbst einer vorbereitenden Abwicklungsbefugnis genau wissen, welches Abwicklungsinstrument in der Folge zur Anwendung gelangen soll, was sich als in der Realität unmöglich zu erfüllende Forderung erweisen dürfte. Somit steht bzw. stand es durchaus mit den Vorgaben der Richtlinie in Einklang, dass die FMA das Moratorium als Ausübung einer Abwicklungsbefugnis dazu nutzt(e), Zeit zu gewinnen, auch wenn dieses Vorgehen nicht unmittelbar die Anwendung eines Abwicklungsinstruments fördern sollte, sondern „nur“ die Prüfung der Möglichkeiten erleichtert.

Im Ergebnis spricht daher alles dafür, das Moratorium als Abwicklungsmaßnahme im Sinne der RL 2014/59/EU einzustufen und es als Ausübung einer typischen Abwicklungsbefugnis einer Abwicklungsbehörde anzusehen.

\section{Zur Einstufung des Moratoriums als Sanierungsmaßnahme im Sinne der RL 2001/24/EG}

Schließt man - entgegen der hier vertretenen Ansicht - die Anwendung der RL 2014/59/EU auf den Fall der HETA und somit auch auf das von der FMA per Bescheid ausgesprochene Moratorium aus, stellte sich die Frage, ob das Moratorium als Sanierungsmaßnahme gemäß der RL 2001/24/EG eingestuft werden könnte.

Art. 2 siebter Spiegelstrich RL 2001/24/EG definiert Sanierungsmaßnahmen als jene Maßnahmen, „mit denen die finanzielle Lage eines Kreditinstituts gesichert oder wiederhergestellt werden soll und die die bestehenden Rechte Dritter beeinträchtigen könnten“. Wie zuvor erörtert, ${ }^{153}$ ist für die Einstufung einer Maßnahme als Sanierungsmaßnahme im Sinne der RL 2001/24/EG a.F. entscheidend, ob eine Maßnahme von der Zielsetzung getragen ist, die finanzielle Lage eines Institutes direkt oder indirekt zu stabilisieren oder zu verbessern; bei einer Abwicklungsmaßnahme geht es hingegen primär darum, ein Institut mit möglichst geringen Folgewirkungen abzubauen .

Im Fall des Moratoriums der FMA könnte der Aufschub der Zahlungsfrist in erster Linie im Zusammenhang mit der Abwicklung der HETA zu sehen und zu verstehen sein, was für das Vorliegen einer Abwicklungsmaßnahme im Sinne der RL 2014/59/EU sprechen könnte. Ähnlich wie das GSA $^{154}$ wäre das Moratorium damit nicht als Sanierungsmaßnahme einzustufen.

Die besseren Gründe dürften jedoch gegen einen solchen Ansatz sprechen: So ist bereits der Zusammenhang zwischen dem Moratorium und den anzuwendenden Abwicklungsinstrumenten nicht ähnlich unmittelbar wie beim GSA. ${ }^{155}$ Geht bzw. ginge man von der Unanwendbarkeit der RL 2014/59/EU aus, bleibt bzw. bliebe das Moratorium als

151 Vgl. etwa Art. 63 Abs. 1 Bst. d RL 2014/59/EU zur Befugnis, „Rechte, Vermögenswerte und Verbindlichkeiten eines in Abwicklung befindlichen Instituts auf ein anderes Unternehmen $\mathrm{zu}$ übertragen, soweit das andere Unternehmen dem zustimmt". Vgl. etwa Art. 63 Abs. 1 Bst. 1 RL 2014/59/EU zur Befugnis, „das Leitungsorgan und die Geschäftsleitung eines in Abwicklung befindlichen Instituts zu entlassen bzw. zu ersetzen“.

153 Siehe insbesondere B.III.2.a.bb.

154 Siehe hierzu B.III.2.b.bb.

155 S. schon oben C.I. im Zusammenhang mit der Einstufung des Moratoriums als Ausübung einer Abwicklungsbefugnis. 
eigenständige Maßnahme bestehen. Ein reiner Aufschub des Fälligkeitsdatums von Verbindlichkeiten richtet sich in seiner Zielsetzung jedoch klar auf die Sicherung der finanziellen Lage eines Institutes und wenn überhaupt nur indirekt - durch die gewonnene Zeit - als Vorbereitungsmaßnahme auf eine Abwicklung. Auch die Begründung des entsprechenden Bescheides durch die FMA spricht von der Verhinderung des finanziellen Ausblutens der HETA und anderer Schäden, die durch Verhandlungen unter Zeitdruck entstehen könnten.

Hinzu kommt, dass bereits Art. 2 siebter Spiegelstrich RL 2001/24/EG bei der Definition von Sanierungsmaßnahmen als Beispiel unter anderem „Maßnahmen, die eine Aussetzung der Zahlungen erlauben“, nennt. Der Begriff der „Aussetzung“ weist bereits auf ein zeitlich begrenztes Verschieben etwa eines Fälligkeitsdatums von Verbindlichkeiten hin. Nur schwer begründbar schiene es in diesem Lichte, das von der FMA per Bescheid ausgesprochene Moratorium von dieser Definition von Sanierungsmaßnahmen auszuschließen.

Somit kann letztlich das Moratorium, selbst wenn die RL 2014/59/EU für nicht anwendbar befunden wird, als Sanierungsmaßnahme im Sinne der RL 2001/24/EG eingestuft werden. Es kommt ihm somit alternativ zur hier vertretenen Anwendung der RL 2014/59/EU ${ }^{156}$ auch unter Zugrundelegung dieses Ansatzes als Sanierungsmaßnahme nach altem Begriff ${ }^{157}$ die Rechtswirkung des Art. 3 Abs. 2 RL 2001/24/EG zu.

\section{Zusammenfassung}

Die Ergebnisse des vorliegenden Beitrags lassen sich nunmehr in Thesenform wie folgt zusammenfassen.

- Das Vorgehen des österreichischen Gesetzgebers bei der vorzeitigen Anwendung der Vorgaben der RL 2014/59/EU (indem er das GSA erließ und die (teilweise) Anwendbarkeit des BaSAG auf die HETA als Abbaueinheit vorsah) war aus unionsrechtlicher Sicht zulässig. Es stand weder im Widerspruch zur vor Ablauf der Umsetzungsfrist der RL 2014/59/EU geltenden Rechtslage, noch zu den Verpflichtungen vor Ablauf der Umsetzungsfrist der Richtlinie, noch $\mathrm{zu}$ den Bestimmungen der Richtlinie selbst.

- Darüber hinausgehend ergibt sich bei näherer Prüfung aus dem Unionsrecht sogar eine Verpflichtung für den Gesetzgeber zu einem solchen Vorgehen. Die Untersuchung des persönlichen Anwendungsbereichs der RL 2014/59/EU und insbesondere die Erörterung des Begriffs des Instituts im Sinne der Richtlinie zeigen nämlich, dass nur eine Auslegung, die den Prozesscharakter von Abwicklungsvorgängen angemessen berücksichtigt, zu überzeugen vermag. Zwar wäre auch eine statische Auslegung, die für den Begriff des Instituts, das in den Anwendungsbereich der RL 2014/59/EU fällt, nur auf den Zeitpunkt des Wegfalls der Zulassung abstellt, a priori denkbar. Jedoch spricht bei Anwendung von auf den Wortlaut gestützten, teleologischen und systematischen Auslegungsmethoden sowie einer dogmatisch auf die Sperrwirkung der Richtlinie abgestützten Folgenabschätzung eine Vielzahl von Gründen gegen eine solche Auslegung.

- Daraus ergibt sich eine Verpflichtung für den mitgliedstaatlichen Gesetzgeber, bei der Umsetzung der Richtlinie in das nationale Recht sicherzustellen, dass auch solche Unternehmen in den Anwendungsbereich der RL 2014/59/EU einbezogen werden, bei

156 C.I.

157 Siehe hierzu bereits B.III.2.b.bb. 
denen erste Schritte eines Abwicklungsprozesses bereits vor Ablauf der Umsetzungsfrist der Richtlinie erfolgt sind, die deswegen ihre Zulassung bereits vor Ablauf der Umsetzungsfrist der Richtlinie verloren haben, und deren Situation zugleich eine typische Gefahrenlage schafft, auf deren Bewältigung die Richtlinie abzielt. Da diese Voraussetzungen im Fall der HETA zu bejahen sein dürften, entsprach der österreichische Gesetzgeber somit den Vorgaben der Richtlinie 2014/59/EU, indem er durch das GSA die Schaffung einer Abbaueinheit unter bereits der Richtlinie entsprechenden Vorgaben sicherstellte und ab Ablaufen der Umsetzungsfrist der Richtlinie im BaSAG die Anwendung der Richtlinienvorgaben auf die HETA erneut ausdrücklich sicherstellte.

- $\quad$ Festzuhalten ist allerdings auch, dass die volle Rechtswirkung der RL 2014/59/EU erst am 1.1.2015 einsetzte und somit auch konkret getroffene Abwicklungsmaßnahmen erst ab diesem Datum der Richtlinie unterliegen bzw. auch dementsprechend unionsrechtlich determinierte Rechtswirkungen entfalten.

- $\quad$ Beim GSA handelt es sich inhaltlich um eine Abwicklungsmaßnahme im Sinne der RL 2014/59/EU und somit für den Fall der als erforderlich erachteten Schaffung der Abbaueinheit HETA tatsächlich um eine vorweggenommene Umsetzung der RL 2014/59/EU. Nicht erforderlich ist hierfür, dass sämtliche Aspekte des Gesetzes unter die Richtlinie subsumiert werden können, da diese keine Vollharmonisierung vorsieht und den Mitgliedstaaten die Schaffung zusätzlicher Abwicklungsinstrumente ermöglicht. Das GSA umfasst inhaltlich betrachtet schwerpunktmäßig die Anwendung der Abwicklungsinstrumente der Unternehmensveräußerung und der Ausgliederung von Vermögenswerten im Sinne der RL 2014/59/EU. Zu beachten sind an dieser Stelle die zuvor genannten zeitlich begrenzten Rechtswirkungen, die eine solche Einstufung unter die Richtlinie nach sich ziehen, da diese erst ab Ablauf der Umsetzungsfrist der Richtlinie einsetzen können.

- Untersucht man den Anwendungsbereich bzw. dessen Änderungen bei den RL 2001/24/EG und 2014/59/EU näher, zeigt sich, dass weiterhin zur Feststellung des Anwendungsbereichs der RL 2001/24/EG zuerst auf den bereits in der alten Fassung vorhandenen Begriff der Sanierungsmaßnahmen abzustellen ist. Sanierungsmaßnahmen in diesem Sinne zielen auf die Sicherung oder Wiederherstellung der finanziellen Lage eines Instituts ab. Die RL 2014/59/EU fügt diesem Begriff der Sanierungsmaßnahmen die Kategorie der Abwicklungsmaßnahmen aus der RL 2014/59/EU hinzu, die nunmehr als Teil eines erweiterten Begriffes der Sanierungsmaßnahmen zu verstehen sind. All diesen Sanierungsmaßnahmen im weiteren Sinn kommen die in RL 2001/24/EG vorgesehenen Rechtswirkungen $\mathrm{zu}$, was insbesondere die Anerkennungspflicht in anderen Mitgliedstaaten nach Art. 3 Abs. 2 RL 2001/24/EG betrifft.

- Der Begriff der Sanierungsmaßnahmen in der RL 2001/24/EG ist weit zu verstehen. Dessen ungeachtet zeigt sich bei einer Prüfung der einzelnen Vorgaben des GSA, dass diese sich weitgehend nicht unter diesen Begriff fassen lassen, da sie primär auf die Abwicklung und nicht die Sicherung der Hypo Alpe-Adria-Bank International AG gerichtet sind. Eine Ausnahme könnte die Übertragung von Anteilen der Bank in eine eigene Aktiengesellschaft darstellen; dies wäre jedoch noch näher - auch ökonomisch - zu prüfen bzw. zu begründen.

- Das per Bescheid vom 1. März 2015 ausgesprochene Moratorium der FMA lässt sich soweit man die Anwendbarkeit der RL 2014/59/EU auf den Fall der HETA grundsätzlich bejaht - eindeutig als Ausübung einer Abwicklungsbefugnis und somit als Abwicklungsmaßnahme einstufen. Eine weitere Prüfung zeigt, dass auch für den Fall, dass die RL 2014/59/EU für nicht anwendbar befunden wird, das Moratorium wohl 
als Sanierungsmaßnahme im Sinne der RL 2001/24/EG einzustufen ist und ihm auf dieser Grundlage ebenfalls die Rechtswirkungen des Art. 3 Abs. 2 RL 2001/24/EG zukommen, was insbesondere eine Anerkennungspflicht für andere Mitgliedstaaten mit sich bringt. 